\title{
Multiple solutions, Oscillons and Strange Attractors in Thermoviscoelastic Marangoni Convection
}

\author{
Marcello Lappa ${ }^{1}$ and Hermes Ferialdi
}

\author{
${ }^{1}$ Department of Mechanical and Aerospace Engineering, University of Strathclyde, James Weir \\ Building, 75 Montrose Street, Glasgow, G1 1XJ, UK - email: marcello.lappa@strath.ac.uk
}

\begin{abstract}
Through numerical solution of the governing time-dependent and non-linear NavierStokes equations cast in the framework of the Oldroyd-B model, the supercritical states of thermal Marangoni-Bénard convection in a viscoelastic fluid are investigated for increasing values of the relaxation time while keeping fixed other parameters (the total viscosity of the fluid, the Prandtl number and the intensity of the driving force, $\mathrm{Ma}=300$ ). A kaleidoscope of patterns is obtained revealing the coexistence of different branches of steady and oscillatory states in the space of parameters in the form of multiple solutions. In particular, two main families of well-defined attractors are identified, i.e. multicellular steady states and oscillatory solutions. While the former are similar for appearance and dynamics to those typically produced by thermogravitational hydrodynamic disturbances in layers of liquid metals, the latter display waveforms ranging from pervasive standing waves to different types of spatially localised oscillatory structures (oscillons). On the one hand, these localised phenomena contribute to increase the multiplicity of solutions and, on the other hand, give rise to interesting events, including transition to chaos and phenomena of intermittency. In some intervals of the elasticity number, the interference among states corresponding to different branches produces strange attractors for which we estimate the correlation dimension by means of the algorithm originally proposed by Grassberger and Procaccia.
\end{abstract}

Key words: Oscillons, multiple solutions, strange attractors, thermal convection.

\section{Introduction}

As discussed by Bergè et al. ${ }^{1}$, the evolution of a dynamical system can be described by means of a phase trajectory, which is a curve traced in the phase space having as many dimensions as the number of degrees of freedom of the system. A framework for understanding such dynamics is represented by the so-called chaos theory, a modern development standing at the intersection of physics and mathematics. In the last 30 years, certain universal routes, which dissipative systems take in transitioning from regular to irregular states, have been identified, including (but not limited to) the Ruelle-Takens scenario, the Feigenbaum scenario, and the Manneville-Pomeau scenario. The field continues to burgeon and bring surprises to this day (not all of the potential paths of evolution and related intricacies have been discovered or observed yet). Leaving aside for a while the related differences, such scenarios share a feature, that is, they predict the evolution towards chaos due to the excitation of new degrees of freedom in the considered system ${ }^{1}$.

A typical workhorse to test these theories is represented by thermal convection: The study of pattern formation in thermal convection is an extremely vast field encompassing different "variants" such 
as thermogravitational (Bontoux et al., ${ }^{2}$ ), thermocapillary (Imaishi et al., ${ }^{3}$; Shevtsova et al., ${ }^{4,5}$; Lappa $^{6,7}$; Gaponenko et al., ${ }^{8}$ ) and thermovibrational flows (Mialdun et al., ${ }^{9}$ ).

As an example, for the case of gravitational Rayleigh-Bénard (RB) flow, a fairly comprehensive picture has been elaborated in the last three or four decades. These studies include the pattern formation in layers of infinite extent (formulated in the Busse's extensive theory), the discovery of spiral-defect chaos (Liu and Ahlers ${ }^{10}$ ), transition to turbulence (Ahlers et al., ${ }^{11}$, Chillà and Schumacher ${ }^{12}$ ), the dynamics of systems transversely heated, the joint influence of vertical and horizontal temperature differences (inclined layer convection, see, e.g., Lappa and Gradisncak ${ }^{13}$ ), natural flows in nanofluids (Saghir et al., ${ }^{14}$ ) and so on (see, e.g., Lappa ${ }^{15,16}$ for a recent review). For the specific case of liquid metals and systems being heated from the side (temperature gradient perpendicular to gravity), interestingly, a running line of inquiry has entirely been devoted to the ability of these systems to produce multiple solutions (see, e.g., Crespo del Arco et al., ${ }^{17}$; Pulicani et al., ${ }^{18}$; Gelfgat et al., ${ }^{19}$, Lappa and Ferialdi ${ }^{20}$ ), i.e. to explore the existence in the space of parameters of different branches of laminar flows that coexist and can be effectively selected by a fluiddynamic process depending on its initial state. Interestingly, this property is akin to that of fully turbulent flow for which SIC (sensitivity to initial conditions) is a well-established feature.

Paralleling such an increase in our understanding of dissipative systems such as those represented by the natural fluid-dynamic processes mentioned above, has been the recent identification of some special states, which can be "excited" in granular matter or colloidal suspensions when they are subjected to "vibrations" or other types of harmonic forcing (Clément et al., ${ }^{21}$; Umbanhowar et al., ${ }^{22}$; Tsimring and Aranson, ${ }^{23}$; Lioubashevski et al., ${ }^{24}$ ). These excitations, typically manifesting as localised time-periodic states in an otherwise uniform or patternless background, have been named “oscillons”. Unlike states that display confinement only in the direction perpendicular to the pattern's wave vector (e.g., wormlike solutions such as those reported by Busse and Whitehead ${ }^{25}$; Dennin et al., ${ }^{26}$; Riecke and Granzow ${ }^{27}$ ), oscillons are highly localised in all spatial directions. Rather than producing chaos, these features have typically a propensity to assemble into 'molecular' and 'crystalline' structures forming very regular and ordered patterns (Umbanhowar et al., ${ }^{22}$, Rogers et $\mathrm{al}_{.}{ }^{28}$ ).

In the present work, we identify similar features in a completely different problem in which, for the first time, oscillons, which we loosely define as the spontaneous localization or confinement of oscillatory phenomena to a limited subregion of an otherwise stationary pattern in a translationally invariant system, are seen even if no mechanical forcing is present. More precisely we consider simple surface-tension driven convection in a layer of a viscoelastic fluid uniformly heated from below (i.e. Marangoni-Bénard convection, MB, also known as the Pearson instability after Pearson $^{29}$ ), which we expressly select as a minimal model to inquire about the role of oscillons in determining the states of a dissipative system when new degrees of freedom are progressively excited.

There is a vast literature dedicated to this type of flow and related hierarchy of bifurcations when the control parameter is increased for the case of Newtonian fluids. The interested reader may 
consider, for instance, Schwabe ${ }^{30}$ for recent experiments and Colinet et al., ${ }^{31}$ for an extensive review of existing studies for Newtonian fluids.

For high-Pr fluids it is known that as the disturbances responsible for the onset of convection saturate their amplitude, the flow self-organize to form steady aesthetic hexagonal cells. Other steady patterns, however, become possible if the Marangoni number is increased. They differ from the classical honeycomb structure essentially due to a change in the prevailing topology, which shifts from a hexagon-based symmetry to a new organization based on the coexistence of squares (Bestehorn $^{32}$ ). Interestingly, the transition sequence arises via localised changes by which threefold vertices of the initial hexagonal cells become fourfold. The tendency to develop square cell can also be reinforced by a decrease in the value of the Prandtl number (while keeping fixed the Marangoni number). Indeed, at $\mathrm{Pr}=\mathrm{O}(1)$ the primary mode of $\mathrm{MB}$ convection is given by square cells instead of hexagons. These cells coexist with rolls near the threshold of the primary instability. On increasing Ma, in turn, such structures tend to be replaced by unsteady triangular cells.

If the Prandtl number is further reduced (liquid metals, $\operatorname{Pr}<0.23$ ), as predicted by Thess and Bestehorn $^{33}$ (the reader being also referred to Parmentier et al., ${ }^{34}$ ), steady hexagons are recovered as the preferred mode of convection. Nevertheless, the flow perpendicular to the free surface changes direction with respect to the typical structure seen for $\operatorname{Pr}>1$, which makes such hexagons the mirror image of those generally seen for high Prandtl number liquids, thereby explaining why they are generally called "inverted hexagons”.

Some attempts aimed to clarify the dynamics in the case of viscoelastic fluids are relatively rare and sparse (relevant and valuable examples along these lines being represented by the few works cited in the following). By careful readings of these works, the reader will easily realise that some general consensus exists that when fluid elasticity enters the dynamics, as the well-known principle of exchange of stability is no longer valid, both stationary and oscillatory solutions become possible at the onset of convection. In particular, steady states are taken over by oscillatory solutions when a given threshold in terms of relaxation time is exceeded (Getachew and Rosenblat ${ }^{35}$; Lebon and Cloot $^{36}$; Lebon et al., ${ }^{37}$; Dauby et al., ${ }^{38}$; Siddheshwar et al., ${ }^{39}$ ). In general, the critical Marangoni number required for these oscillatory states to emerge is smaller than the corresponding value for Newtonian fluids. This condition is generally referred to as “overstability”, see, e.g., Ramkissoon et al., ${ }^{40}$ and Hernández Hernández and Dávalos-Orozco, ${ }^{41}$.

Notably, with a few exceptions, where some attempts were made to explore the system response for slightly supercritical conditions (see, e.g., Wilson ${ }^{42}$ and Parmentier et al., ${ }^{43}$, who proposed some numerical experiments which elegantly illustrate weakly non-linear dynamics), existing studies have essentially been limited to the determination of the conditions for the onset of the primary (steady or oscillatory) flow in the framework of the typical protocols of the linear stability analysis. In the present work we expressly target the investigation and ensuing understanding of the system dynamics for conditions in which solution of the complete equations in their non-linear and timedependent form is required. Indeed, we consider circumstances with the Marangoni number attaining magnitudes corresponding to several times the critical value for the Newtonian case. 
Beyond the mere motivation to bridge the abovementioned gap, we are specifically interested in pushing forward the MB system as a new archetypal problem to improve our fundamental understanding of some of the processes discussed before (about the general and specific dynamics of dissipative systems). More precisely, our two-fold purpose is to show that 1) MB convection in viscoelastic fluids can produce oscillons just as vibrated granular matter or colloidal suspensions; 2) apart from supporting the formation of ordered patterns ${ }^{22}$, oscillons and their interactions can even promote transition to chaos (via the alternate route represented by the Curry-and-Yorke scenario, a still debated possible substitute of the Ruelle-Takens scenario, see, e.g. Khayat ${ }^{44}$ and $\mathrm{Li}$ and Khayat ${ }^{45}$ ). Most interestingly, as we will show later, for the present conditions such a mechanism is mediated by the coexistence of "multiple solutions" and the emergence of strange attractors as the relaxation time of the considered viscoelastic fluid is increased.

\section{Mathematical Model}

\subsection{The balance equations}

The balance equations for mass, momentum and energy, can be cast in dimensional form as

$$
\underline{\nabla} \cdot \underline{V}=0
$$

$$
\rho \frac{D \underline{V}}{D t}=\underline{\nabla} \cdot \underline{\tau}+\underline{\nabla} \cdot \underline{\tilde{\tau}}
$$

$$
\frac{\partial T}{\partial}+\underline{\nabla} \cdot[\underline{V T}]=\alpha \nabla^{2} T
$$

Where $\underline{V}$ and $\mathrm{T}$ are the fluid dimensional velocity and temperature ( $\rho$ and $\alpha$ being the fluid density and thermal diffusivity, respectively), $\underline{\underline{\tau}}$ is the so-called viscous (Newtonian) stress tensor and $\underline{\underline{\tau}}$ is the additional tensor accounting for the contribution brought to the physical stresses present in the fluid by viscoelasticity. Indeed viscoelasticity can be defined as the property of a fluid to display both viscous and elastic properties under the same conditions when it is subjected to a deformation.

\subsection{The microscopic characterisation of viscoleastic fluids}

The derivation of macroscopic equations accounting for the viscoelastic stresses and their evolution in time starting from microscopic considerations is not straightforward as one would imagine. The mathematical explanation of a viscoelastic fluid is much more complex than its Newtonian counterpart. Nevertheless, just as in the case of Newtonian fluids, for which it is possible to 
introduce formally the macroscopic balance equation for momentum by starting from a microscopic formulation based on the Newton's law (the interested reader being referred to Lappa ${ }^{15}$ for an exhaustive treatment), it is possible to apply a similar approach to build a macroscopic expression for the viscoelastic (extra) stresses tensor starting from the so-called Dumbbell model.

This approach formally yields an additional macroscopic transport equation (see, e.g., the related derivation given by Revuz and Yor ${ }^{46}$ ) governing the evolution in time of $\underline{\tilde{\tau}}$. This equation reads (Alves et al., ${ }^{47}$ and references therein)

$$
\lambda\left(\frac{\partial \underline{\underline{\tau}}}{\partial}+\underline{V} \cdot \underline{\nabla} \underline{\underline{\tau}}\right)+\underline{\tilde{\tau}}=\eta\left(\underline{\nabla} \underline{V}+\underline{\nabla} \underline{V}^{T}\right)+\lambda\left(\underline{\underline{\tau}} \cdot \underline{\nabla} \underline{V}+\underline{\nabla} \underline{V}^{T} \cdot \underline{\tilde{\tau}}\right)
$$

where $\lambda=\xi / 4 \mathrm{H}$ represents the so-called relaxation time and the viscosity of the polymer $\eta$ can be expressed as $\left(2 \mathrm{n} \lambda H a^{2}\right)=\left(\mathrm{n} \xi a^{2} / 2\right)$, where $\xi$ is the drag coefficient relating to each bead of the Dumbell model (which mimics a molecule as a system formed by two beads connected by a spring, Bird et al., ${ }^{48}$, Bonito et al., ${ }^{49}$ ). Moreover, $\mathrm{H}$ is the spring stiffness or Hook modulus, $\mathrm{n}$ is the concentration of polymer molecules and $a$ is the radius of gyration of the considered molecule. This equation relates to a class of macroscopic viscoelastic models, generally known under the heading of Oldroyd-B.

By formally closing the problem from a mathematical point of view, as it introduces a connection between $\tilde{\tau}$ and the fluid velocity $\underline{V}$, this equation allows a rich variety of fluid-dynamics effects to be enabled (the main object of the present study), which are not possible otherwise (i.e. in standard Newtonian liquids). Along these lines, in the present work we expressly rely on such a framework, such a choice being motivated by the three-fold realisation that 1) for this model, a macroscopic constitutive equation with precise physical meaning can be obtained by exact integration of a relevant stochastic microscopic equation (Bird et al., ${ }^{48}$ ); 2) this model has enjoyed a widespread use in the literature and it is currently considered a kind of paradigm or reference "testbed" for the identification of new thermal convective effects in viscoelastic fluids ( $\mathrm{Li}$ and Khayat ${ }^{45}$; Hu et al., ${ }^{50}$ ); 3) it can adequately represent highly elastic solutions consisting of a Newtonian solvent and a polymeric solute (a relevant example along these lines being a polyacrylamide solution in a maltose syrup/water mixture ${ }^{45}$ ). These solutions, which possess an essentially constant viscosity over a wide range of shear rates, are also known as 'Boger fluids, ${ }^{48}$.

\subsection{The complete set of non-dimensional equations and related boundary conditions}

Taking into account that for an incompressible fluid $\underline{\nabla} \cdot \underline{V}=0$ and

$$
\underline{\nabla} \cdot\left[2(\underline{\nabla} \underline{V})_{o}^{s}\right\rfloor=\underline{\nabla} \cdot\left[\underline{\nabla} \underline{V}+\underline{\nabla} \underline{V}^{T}\right]=\left[\nabla^{2} \underline{V}+\underline{\nabla}(\underline{\nabla} \cdot \underline{V})\right]=\nabla^{2} \underline{V}
$$


scaling velocity, pressure, temperature and the viscoelastic stress by proper reference quantities, namely, $\alpha / \mathrm{d}$ as reference velocity, $\mathrm{d}^{2} / \alpha$ as reference time, $\rho \alpha^{2} / \mathrm{d}$ as reference pressure, $\Delta T$ as reference temperature and $\rho v \alpha / d^{2}$ as reference viscoelastic stress (where $d$ is the depth of the fluid layer, $v$ is the solvent kinematic viscosity and $\alpha$ the fluid thermal diffusivity), the overarching equations for the considered category of problems (solenoidal velocity field) can be cast in nondimensional form as:

$$
\begin{aligned}
& \frac{\partial \underline{V}}{\partial}+\underline{\nabla} \cdot[\underline{V} \underline{V}]=-\underline{\nabla} p+\operatorname{Pr} \nabla^{2} \underline{V}+\operatorname{Pr} \underline{\nabla} \cdot \underline{\underline{\tau}} \\
& \theta\left(\frac{\partial \underline{\underline{\tau}}}{\partial}+\underline{V} \cdot \underline{\nabla} \underline{\underline{\tau}}\right)+\underline{\tilde{\tau}}=\zeta\left(\underline{\nabla} \underline{\underline{V}}+\underline{\nabla} \underline{V^{T}}\right)+\theta\left(\underline{\tilde{\tau}} \cdot \underline{\nabla} \underline{V}+\underline{\nabla} \underline{V}^{T} \cdot \underline{\tilde{\tau}}\right) \\
& \frac{\partial T}{\partial}+\underline{\nabla} \cdot[\underline{V T}]=\nabla^{2} T
\end{aligned}
$$

where a variety of characteristic nondimensional numbers can be easily identified, namely, the classical Prandtl number $\operatorname{Pr}=v / \alpha$, the viscosity ratio $\zeta=\eta / \mu$ (where $\mu$ and $\eta$ are the dynamic viscosities of the solvent and polymer, respectively) and the parameter $\theta=\alpha \lambda / \mathrm{d}^{2}$. This parameter may be regarded as an analogue of the so-called Deborah number, see, e.g., Parmentier et al., ${ }^{43}$, also known as the "elasticity number", Li and Khayat, ${ }^{45}$. Related additional characteristic parameters are $\beta=\mu /(\mu+\eta)$ and the generalised Prandtl number $\operatorname{Pr}_{g}=\operatorname{Pr} / \beta$. Given the assumption of dilute suspension, the addition of polymer to the initial Newtonian liquid is assumed to have a negligible impact on the resulting fluid density.

As anticipated in the introduction, the system considered here is a layer of fluid heated from below (see Fig. 1) with heat exchange at the free (undeformable) interface and periodic boundary conditions at $\mathrm{x}=0\left(0 \leq \mathrm{z} \leq \mathrm{A}_{\mathrm{z}}\right)$ and $\mathrm{x}=\mathrm{A}_{\mathrm{x}}\left(0 \leq \mathrm{z} \leq \mathrm{A}_{\mathrm{z}}\right)$ and at $\mathrm{z}=0\left(0 \leq \mathrm{x} \leq \mathrm{A}_{\mathrm{x}}\right)$ and $\mathrm{z}=\mathrm{A}_{\mathrm{z}}\left(0 \leq \mathrm{x} \leq \mathrm{A}_{\mathrm{x}}\right)$ where $A_{x}=L / d$, $L$ being the horizontal extension of the computational domain; $A_{z}$ is assumed equal to $A_{x}$ for the $3 \mathrm{D}$ simulations or to be infinite under the assumption of 2D flow. The three components of the non-dimensional velocity $\underline{V}$ along the three axes of the Cartesian reference system are denoted by $\mathrm{V}_{\mathrm{x}}, \mathrm{V}_{\mathrm{y}}$ and $\mathrm{V}_{\mathrm{z}}$, respectively.

As we do not consider buoyancy in our model, we deal with "pure" surface-tension driven flows. A review of works about the surface tension of polymer liquids is due to Dee and Sauer ${ }^{51}$, where it is shown that the surface tension for polymer molecules, in general, is assumed to be linear with the temperature as in the case of Newtonian fluids. The presence of surface-tension driven stresses at the free surface in the present problem, therefore, implies the following kinematic boundary condition:

$$
\left\lfloor 2(\underline{\nabla} \underline{V})_{o}^{s}+\underline{\tilde{\tau}}\right\rfloor \cdot \underline{\hat{n}}=-M a(\underline{\underline{I}}-\underline{\hat{n}} \underline{\hat{n}}) \cdot \underline{\nabla T}
$$


where $\underline{\hat{n}}$ is the unit vector perpendicular to the liquid/gas interface (directed from liquid to gas), $\underline{\underline{I}}$ is the unity matrix and $\mathrm{Ma}$ is the so-called Marangoni number $\mathrm{Ma}=\sigma_{\mathrm{T}} \Delta \mathrm{Td} / \mu \alpha$, $\sigma_{\mathrm{T}}$ being the derivative of surface tension with respect to temperature.

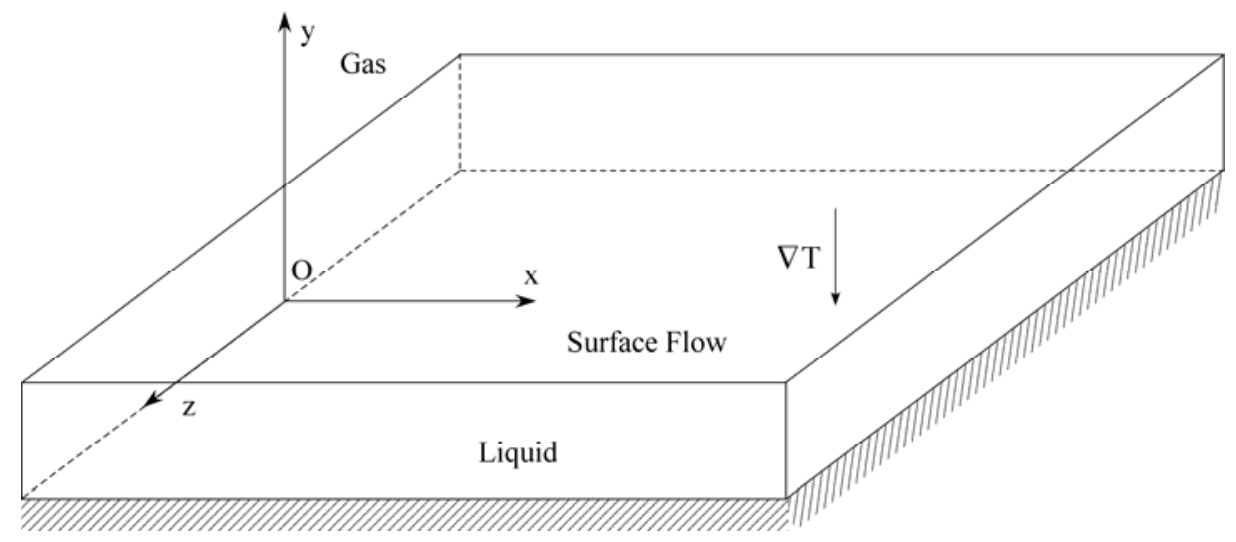

Figure 1: Sketch of the considered problem

A fixed temperature is imposed on the bottom (no-slip) wall, with the temperature at the free surface satisfying the condition:

$\underline{\nabla T} \cdot \hat{n}=-B i T$

where $\mathrm{Bi}$ is the well-known Biot number (classically defined as $\mathrm{Bi}=\mathrm{hd} / \mathrm{k}$ where $\mathrm{h}$ and $\mathrm{k}$ are the gas convective heat transfer coefficient and the liquid thermal conductivity, respectively).

We do not describe here the initial conditions, as this point is discussed directly in the section of results owing to its crucial role in producing multiple solutions. All flow frequencies reported in Sect. 4 have been made non-dimensional using as reference frequency the inverse of reference time $\left(\alpha / d^{2}\right)$.

\section{Numerical Method}

The set of overarching equations and related boundary conditions have been integrated numerically using the OpenFoam computational platform. For incompressible fluids, this platform relies on the so-called category of projection methods. These methods stem from a well-known property of the momentum equation, namely its ability to physically retain the vorticity being associated with the considered problem if the pressure gradient is (unphysically) dropped. From a purely mathematical point of view, this property is directly connected to the irrotational nature of every vector field 
resulting from the gradient of a scalar quantity (the pressure gradient in this case), which therefore does not contribute to the curl of the velocity field.

By integrating the momentum equation deprived of the pressure gradient, an intermediate velocity field is obtained, which, however, is not solenoidal. This leads to the need to correct it to make the resulting field incompressible. This is implemented by defining a new velocity field as a linear combination of the earlier (intermediate) field and the pressure gradient previously disregarded. As in such a stage the pressure plays the role of an unknown, an additional equation needs to be solved to determine it. This equation is formally obtained by forcing the "corrected" velocity field into the continuity equation. This produces a purely elliptic equation, which unlike the momentum equation (parabolic in nature with respect to time and elliptic with respect to space) has to be solved iteratively (see, e.g., Lappa ${ }^{52}$ ).

Different variants of such approach are possible according to the philosophy used to integrate the simplified momentum equation with respect to time (via explicit or implicit schemes). We have used the "Pressure Implicit with Splitting Operator", i.e. the PISO approach (see e.g., Jang et al., ${ }^{53}$ and Moukalled et al., ${ }^{54}$ ) implemented in OpenFoam via the so-called Rhie and Chow, ${ }^{55}$ interpolation stencil .

Moreover, the diffusive and convective terms in the parabolic (momentum and energy) equations have been treated with standard central differences and second-order accurate Lax-Wendroff spatial schemes, respectively.

Due to the lack of diffusive terms, which would make it parabolic in time or elliptic in space, unfortunately, the constitutive equation for the viscoelastic stresses is hyperbolic in nature. For this reason, in order to mitigate problems relating to the numerical stability of the algorithm, for this equation the Lax-Wendroff schemes have been replaced with upwind schemes. This approach proved successful over the entire range of considered values of the elasticity number.

Some special attention has been devoted to the momentum equation as well. Following Favero et al., ${ }^{56}$, its numerical stability has been increased by introducing two diffusive terms having similar computational "weight" and meaning, one at the left hand side and the other at the right hand side of the equation. With these two mathematically equivalent contributions, one being numerically implemented in an implicit way, while the other is treated explicitly, the enriched equation reads:

$$
\frac{\partial \underline{V}}{\partial t}+\underline{\nabla} \cdot[\underline{V} \underline{V}]-\operatorname{Pr}(1+\zeta) \nabla^{2} \underline{V}=-\underline{\nabla} p-\operatorname{Pr} \zeta \nabla^{2} \underline{V}+\operatorname{Pr} \underline{\nabla} \cdot \underline{\tilde{\tau}}
$$

Though the two terms are equivalent from a mathematical point of view, they are not from a numerical point of view, which provides the solution with an amount (quantitatively negligible) of residual diffusion sufficient to increase appreciably the "ellipticity" of the momentum equation and improve its numerical stability. 


\section{$\underline{3.1 \text { Validation }}$}

Toward the end of using such an algorithm to address the fundamental questions set in the introduction, it is extremely important that it is validated. In this regard, we have examined a relatively wide spectrum of conditions through comparison with well-established results and other available (relevant) solutions for both cases of isothermal and non-isothermal flows.

In particular, as a first-level activity to test the response of the current implementation of the Oldroyd-B model, we have focused on the classical problem relating to the non-inertial flow through a 4:1 planar contraction (set in the benchmark study by Alves et al., ${ }^{47}$ ). The outcomes of such comparisons are summarised in Table I, from which it can easily be verified that the discrepancy between the present results and the very accurate data reported in that study lie below $2 \%$.

Table I: Comparison with the data reported in the benchmark study by Alves et al. ${ }^{47}$. Variation of the size of vortex $\left(\mathrm{X}_{\mathrm{r}}\right)$ formed in the corner of the contraction with the Deborah number ${ }^{47}$ (De) for the Oldroyd-B fluid (present mesh: $2 \times 10^{4}$ cells).

\begin{tabular}{lll}
\hline \hline De & $\mathrm{X}_{\mathrm{r}}\left[\mathrm{Ref}^{47}\right]$ & $\mathrm{X}_{\mathrm{r}}$ [Present $]$ \\
\hline \hline 0.5 & 1.452 & 1.479 \\
1.0 & 1.279 & 1.301 \\
\hline \hline
\end{tabular}

As a second-level of such a validation hierarchy, after sifting through existing studies in the literature we have considered the data available in the linear stability analysis by Parmentier et al., ${ }^{43}$ for $\operatorname{Pr}_{\mathrm{g}}=1$; this is the most relevant case we could identify with respect to the subjects addressed in the present work.

Table II: Comparison with the results (predicted angular frequency) of the linear stability analysis by Parmentier et al., ${ }^{43} \cdot \operatorname{Pr}_{\mathrm{g}}=1, \theta=0.2, \mathrm{Bi}=0$.

\begin{tabular}{clcl}
\hline \hline$\beta$ & $\varepsilon$ & $\omega\left[\operatorname{Ref}^{43}\right]$ & $\omega[$ Present $]$ \\
\hline \hline 0.1 & 0.792 & 7.4 & 7.3 \\
0.2 & 0.96 & 5.0 & 4.83 \\
\hline \hline
\end{tabular}

As shown in Table II, in particular, we have assessed the response of our algorithm with respect to two different values of the parameter $\beta$ (measuring the relative importance of the solvent and mixture viscosity) and different flow supercriticalities (via the parameter $\varepsilon=\mathrm{Ma} / \mathrm{Ma}_{\mathrm{cr}}$, where $\mathrm{Ma}_{\mathrm{cr}}$ is the critical value for Newtonian fluids).

More precisely, our approach has been based on the following procedure: eqs. (6-8) have been solved numerically for values of $\varepsilon$ slightly larger than those considered by the linear stability 
analysis using a mesh with 200 grid points along the horizontal extension of the layer and 40 points along the vertical direction (see Sect. 3.2 for relevant information about the problem sensitivity to the mesh resolution); then the obtained angular frequencies $(\omega)$ of the oscillatory flow have been extrapolated to the critical value of $\varepsilon$ predicted by the linear stability analysis. In this way, we could obtain for $\beta=0.1$ a value of $\omega=7.3$ matching with a reasonable approximation (less than $1.5 \%$ ) those determined by Parmentier et al., ${ }^{43}$. Similarly for $\beta=0.2$, we found a percentage difference limited to $3 \%$.

\subsection{Mesh refinement study}

The influence of the number of grid points on the numerical solution can be gathered from Tables III and IV for two representative cases corresponding to the onset of stationary and oscillatory convection, respectively. For such simulations, the value of the Marangoni number has been fixed to 300 as for all the other cases considered in the present work (Sect. 4). Starting from initial thermally diffusive conditions, it can be seen that the numerical solution tends towards a welldefined state as the mesh density is increased.

Table III: Influence of mesh on the maximum (non-dimensional) absolute values of the streamfunction $\left(\operatorname{Pr}_{\mathrm{g}}=1, \beta=1 / 5, \mathrm{Ma}=300, \mathrm{Bi}=0.1, \theta=5 \times 10^{-3}\right)$.

\begin{tabular}{c|c|c}
\hline \hline Grid $\mathrm{N}_{\mathrm{x}} \times \mathrm{N}_{\mathrm{y}}$ & $\psi_{\max }$ & \\
\hline \hline $160 \times 32$ & 2.240 \\
$180 \times 36$ & 2.243 \\
$200 \times 40$ & 2.249 \\
$250 \times 50$ & 2.249 \\
\hline \hline
\end{tabular}

Table IV: Influence of mesh on the angular (non-dimensional) frequency $\omega\left(\operatorname{Pr}_{\mathrm{g}}=1, \beta=1 / 5, \mathrm{Ma}=300\right.$, $\mathrm{Bi}=0.1, \theta=1.5 \times 10^{-2}$ ).

\begin{tabular}{rl}
\hline \hline Grid $\mathrm{N}_{\mathrm{x}} \times \mathrm{N}_{\mathrm{y}}$ & $\omega$ \\
\hline $160 \times 32$ & 0.772 \\
$180 \times 36$ & 0.798 \\
$200 \times 40$ & 0.814 \\
$220 \times 44$ & 0.827 \\
$250 \times 50$ & 0.831 \\
\hline \hline
\end{tabular}

It is evident that the percentage difference displayed by the maximum of the streamfunction $\left|\psi_{\max }\right|$ and the angular (non-dimensional) frequency $\omega$ falls below $2 \%$ as soon as the mesh density exceeds that corresponding to the 200x40 resolution. Accordingly we have used 40 points along the vertical direction (y) and 200 points along the horizontal direction (200 points along $\mathrm{x}$ and 200 points along $\mathrm{z}$ leading to a total of $1.6 \times 10^{6}$ grid nodes in the case of three-dimensional simulations). 


\section{Results}

In order to investigate the sensitivity of the system with respect to a single control parameter, i.e. $\theta$, we have fixed $\operatorname{Pr}_{\mathrm{g}}=1, \beta=1 / 5$ ( $\zeta=4$ ), $\mathrm{Ma}=300, \mathrm{Bi}=0.1$, with $\theta$ spanning the interval $0 \leq \theta \leq 0.1$. The considered value of Ma corresponds to approximately four times $\mathrm{Ma}_{\mathrm{cr}}$ for an unbounded layer of a Newtonian fluid. As implicitly defined by the first two conditions, the considered fluid would reduce to a standard Newtonian liquid with $\operatorname{Pr}=1$ in the limit as $\theta \rightarrow 0$ (which is equivalent to setting $\eta=0^{45}$ ). Fluids with $\operatorname{Pr}_{\mathrm{g}} \cong \mathrm{O}(1)$ (although Pr is not exactly $\cong 1$ ) are representative of a class of water-based polymer dilute solutions at relatively high temperatures, e.g., water at $70{ }^{\circ} \mathrm{C}$ with limited amount of a polymer such a PAM, PEG, PEO, PVP, xanthan gum, etc. Assuming $\lambda \cong 10^{-3} \mathrm{~s}$ (a typical realistic value for small polymer concentrations), this value would correspond to $\theta=\alpha \lambda / \mathrm{d}^{2}$ spanning the range from $\cong 5 \times 10^{-3}$ to 0.05 on varying the depth of the layer from $0.5 \mathrm{~mm}$ to $0.05 \mathrm{~mm}$. Though in the initial part of this section we limit ourselves to discussing two-dimensional (2D) findings as a first necessary step towards the interpretation of the even more intricate threedimensional (3D) dynamics, we are obliged by the complexity of the results to articulate their presentation in different stages of discussion (for increasing values of $\theta$ ). In particular, first we address the cases in which the emerging dynamics are clearly recognizable in terms of well-defined attractors in the phase space and then move to more complex situations requiring concepts, methods and tools typical of the chaotic systems analysis.

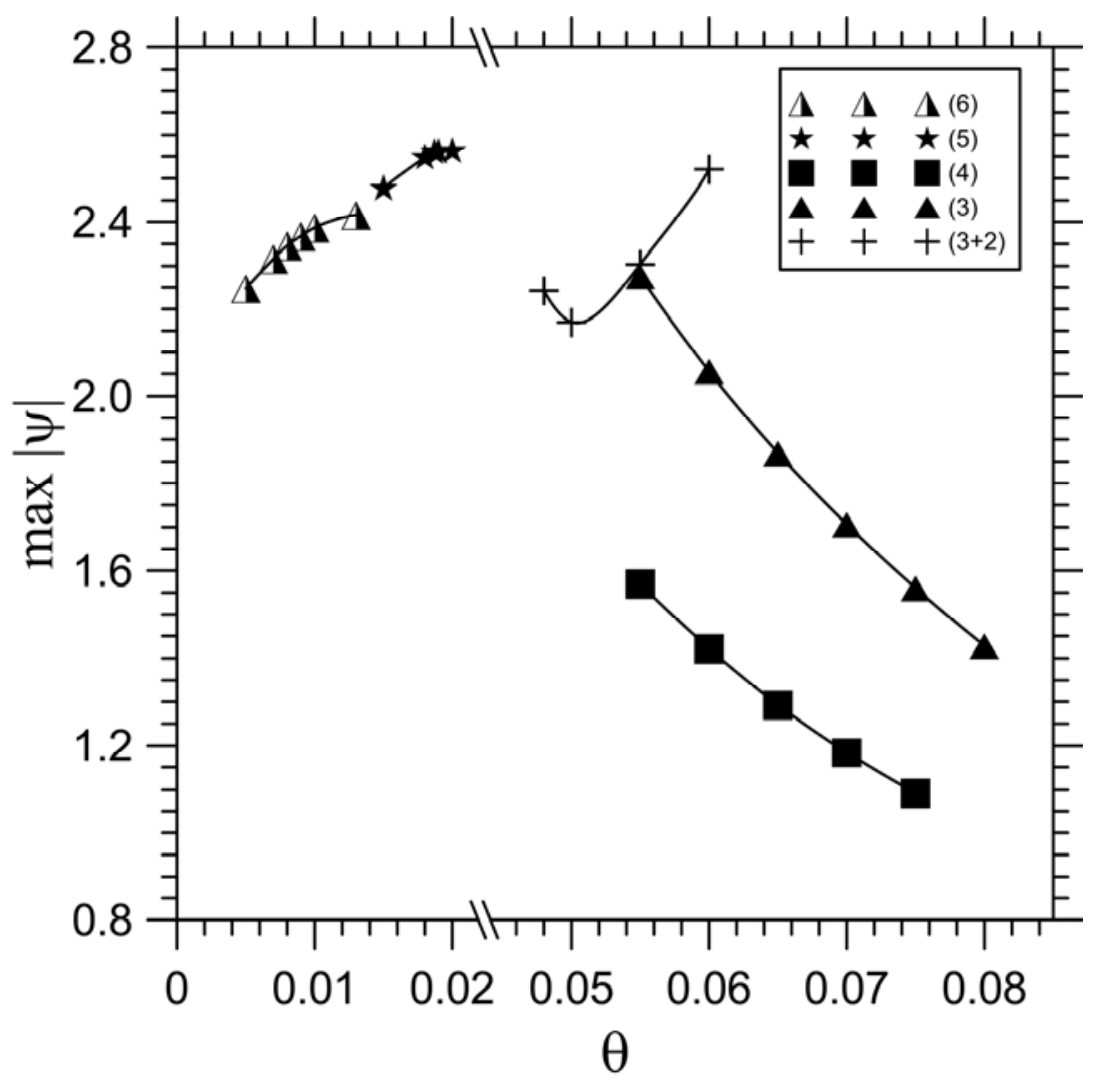

Figure 2: Multiple states of stationary convection in the range $0<\theta \leq 0.08$ (the integer number in round parentheses indicates the couples of counter-rotating rolls present in the system, splines have been used to connect the different points). 


\section{$\underline{4.1 \text { Two-dimensional results and low-dimensional chaos }}$}

As shown in Fig. 2, as soon as $\theta$ is increased starting from the condition of Newtonian fluid $(\theta=0)$, the first striking feature displayed by the problem is the multiplicity of steady solutions in the interval $0<\theta \leq 0.08$.

Indeed, regions of multistability, where the system exhibits up to five disconnected steady-state attractors, can be identified. Such attractors manifest over the considered range of $\theta$ as different branches of solutions (clearly distinguishable in Fig. 2). Though they exist in the full phase space as invariant sets, what we observe in practice (as a result of the numerical simulations), is a projection of these sets onto the physical space, namely multicellular states. These states differ in the extension of the rolls (proportional to the wavelength), the intensity of the emerging flow ( $\left.\psi_{\max }\right)$ and its dependence on $\theta$; indeed, for some branches the maximum value attained by the streamfunction behaves as an increasing function of $\theta$, while for others the opposite behaviour can be seen.

It is known that in a problem developing coexisting branches of solutions, the trajectories of the system can converge selectively to either of the attractors depending on the considered initial conditions. Let us recall that, by definition, the basin of attraction of an attractor is the set of initial points whose trajectories fall on the given attractor. For these reasons, in order to identify the window in which the system is subjected to multiple coexisting attractors, we have used an approach similar to that implemented by Gelfgat et al., ${ }^{19}$ and Lappa and Ferialdi, ${ }^{20}$ for the case thermogravitational flows in transversely heated liquid metals. We have explored the system response when the basin of attraction is varied. More precisely, in addition to the "standard" initial conditions corresponding to a quiescent state, a "forward and backward continuation" method has been used, i.e. the final state at each iteration of the control parameter $\theta$ has been set as the initial state for the next iteration (Kengne et al., ${ }^{57}$ ).

In this way the coexistence of the branches located on the right of Fig. 2 has been identified. As shown in this figure, solutions with either an even (4) or odd (3) number of couples of rolls are possible in the interval $0.055 \leq \theta \leq 0.075$. Interestingly, these regimes also coexist in a more restricted range of $\theta(0.055 \leq \theta \leq 0.06)$ with $(3+2)$ states, which perhaps deserve some additional description.

Indeed, apart from being the only branch for which the maximum of the streamfunction displays a non-monotone behaviour, these solutions also exhibit a remarkable difference in terms of patterning behaviour. This difference (e.g. with respect to the branch with 3 couples of rolls extending from $\theta=$ 0.055 to $\theta=0.08$ ) is due essentially to the presence of two additional couples of counter-rotating rolls with relatively limited strength and spatial extension, which emerge as evenly spaced localised features separating the rolls with larger size and intensity . 


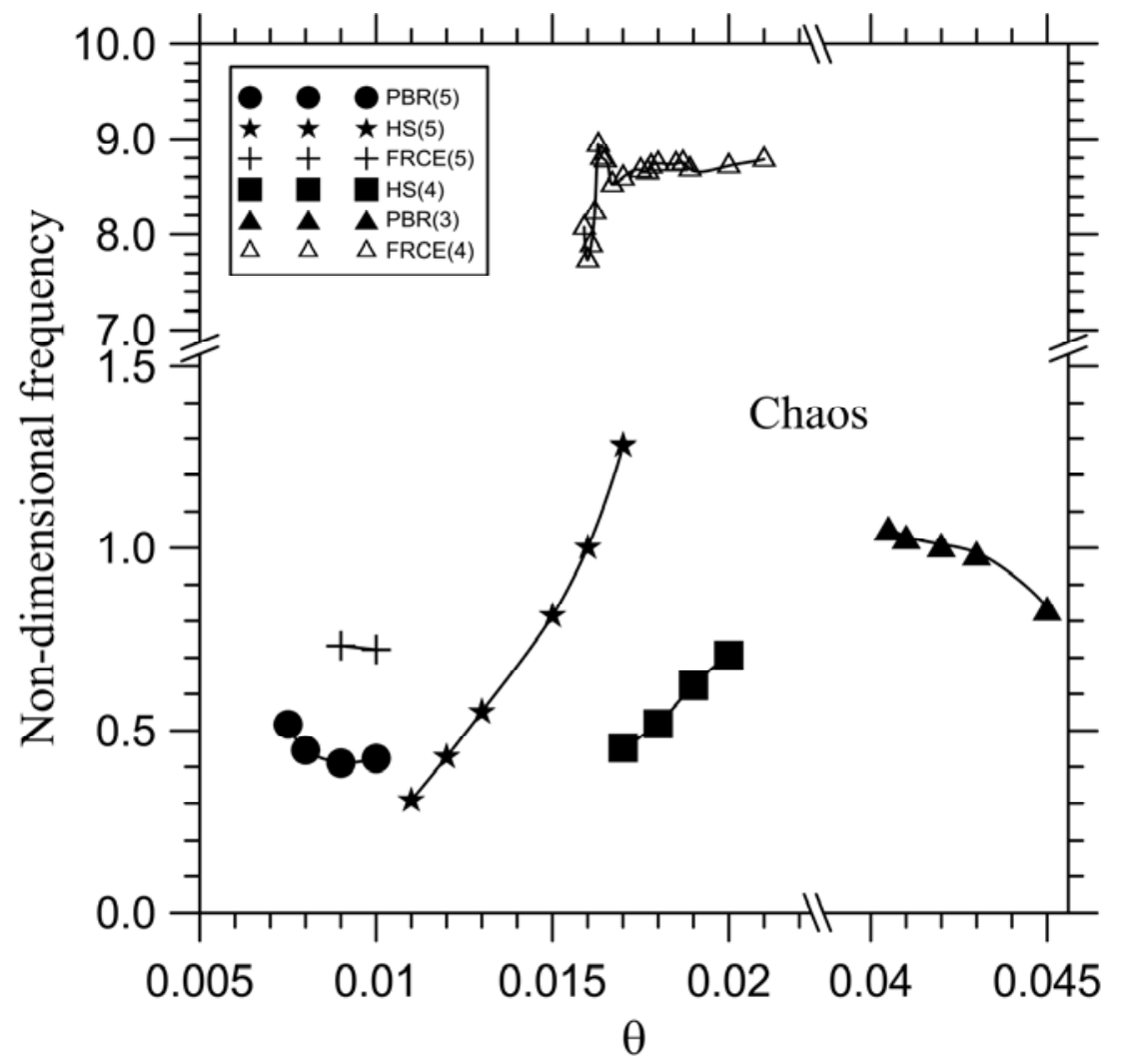

Figure 3: Multiple states of oscillatory convection in the range $0<\theta \leq 0.045$ (Legend: PBR - Purely Bouncing Rolls with fixed shape and size; FRCE - flickering couple of rolls weakly affecting the surrounding stationary state; HS - Hybrid State in which the rhythmic expansion and contraction of a couple of rolls is synchronous with the behaviour of the other rolls, which do not change amplitude but spread periodically along the positive $\mathrm{x}$ axis and then in the opposite direction; the integer number in round parentheses indicates the couples of counter-rotating rolls present in the system).

As summarised in Fig. 3, the just mentioned states of stationary convection with their specific properties even coexist in given sub-intervals of $\theta$ with oscillatory solutions. Some examples are reported in Figs. 4-6 for increasing values of the parameter $\theta$. The fascinating variety of related spatio-temporal behaviours can be summarised as follows: standing waves (in the form of purely constant-amplitude "bouncing” rolls PBR, see e.g., Fig. 6), states with only one periodically contracting and expanding (and/or breaking) couple of counter-rotating rolls in an otherwise almost stationary background flow (localised flickering behaviour, FRCE, see, e.g., Fig. 5) and hybrid states (HS, see e.g., Fig. 4), in which a train of rolls moving horizontally back and forth with constant strength (rolls with fixed shape and size as those typical of the PBR state) is "modulated" by the birth, growth and decay of a localised perturbation. This perturbation leads one of the couples of counter-rotating rolls to grow and shrink in time, i.e. to pulsate in a synchronous manner with respect to the background oscillatory state. 


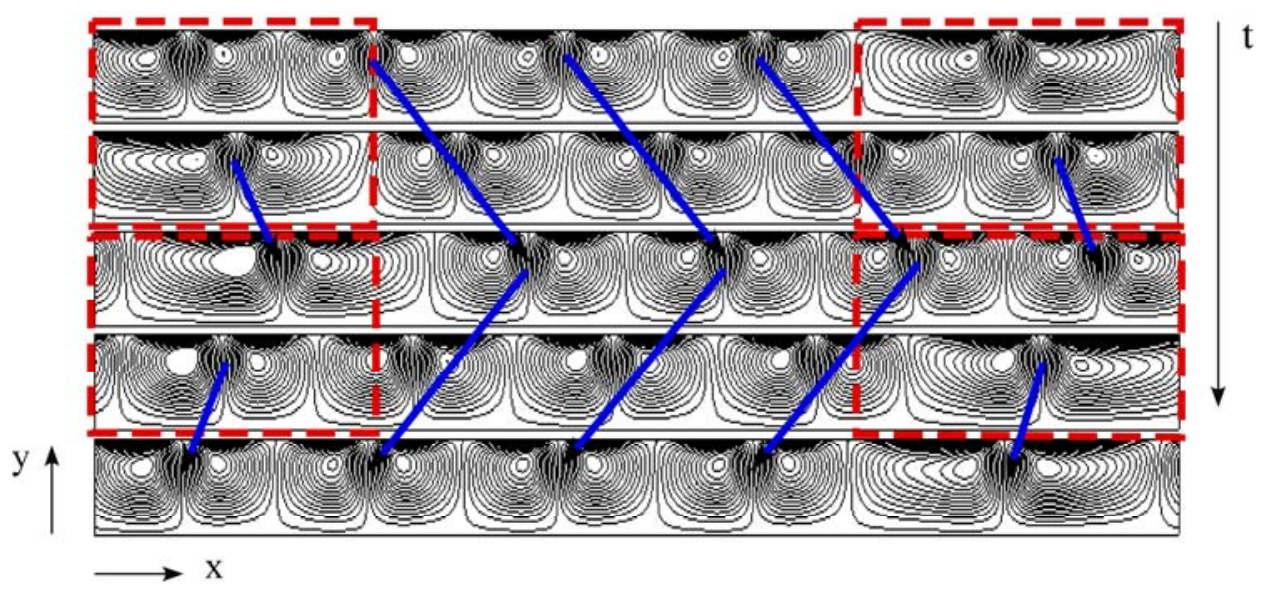

Figure 4: Sequence of snapshots evenly spaced in time revealing typical dynamics for $\theta=0.012$ (HS5).

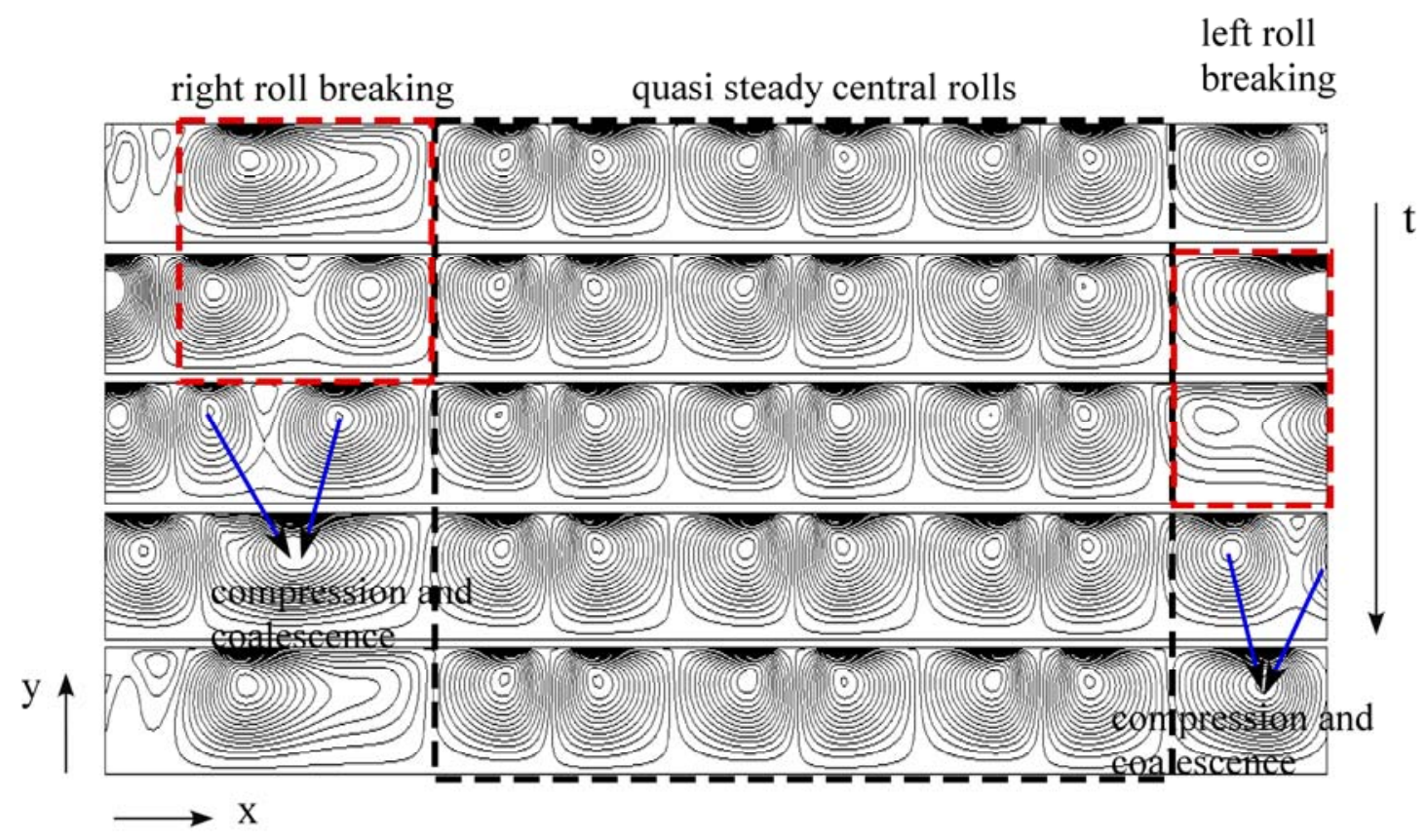

Figure 5: Sequence of snapshots evenly spaced in time revealing typical dynamics for $\theta=0.0175$ (FRCE4). 


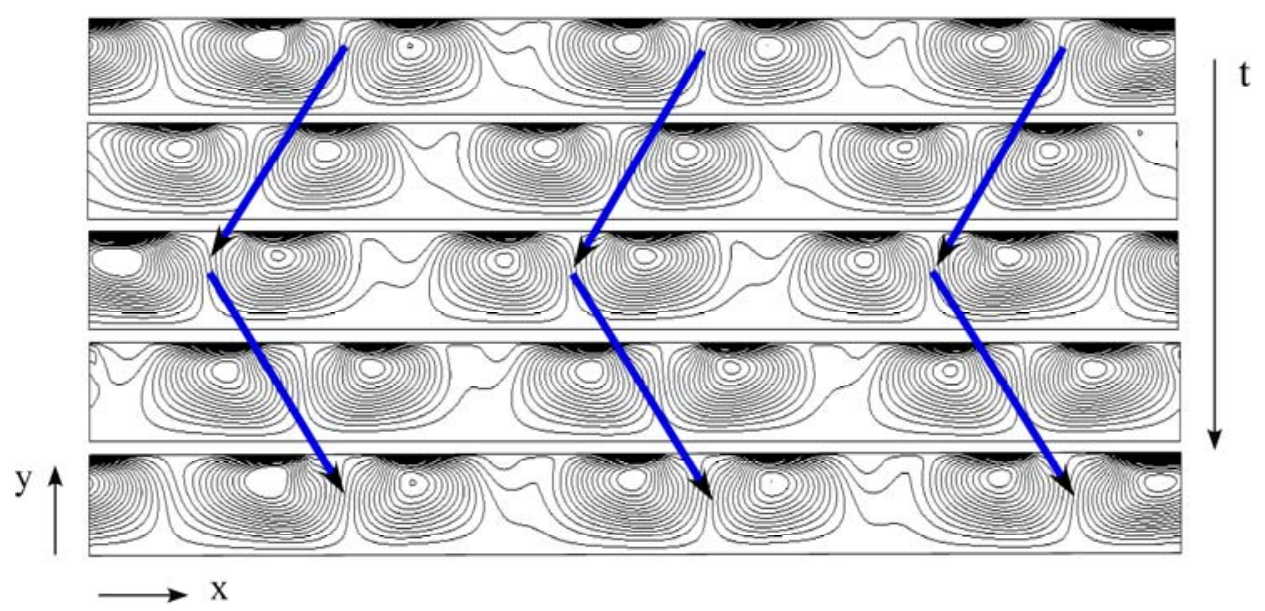

Figure 6: Sequence of snapshots evenly spaced in time revealing typical dynamics for $\theta=0.045$ (PBR3).

The related ranges of existence can be summarised as follows:

Table V: Ranges of existence of the different modes of oscillatory convection

\begin{tabular}{ll}
\hline \hline Elasticity number & Solution \\
\hline \hline $0.007 \leq \theta \leq 0.01$ & PBR(5) \\
$0.009 \leq \theta \leq 0.01$ & FRCE(5) \\
$0.011 \leq \theta \leq 0.017$ & HS(5) \\
$0.016 \leq \theta \leq 0.021$ & FRCE(4) \\
$0.017 \leq \theta \leq 0.0215$ & HS(4) \\
$0.04 \leq \theta \leq 0.048$ & PBR(3) \\
\hline \hline
\end{tabular}

Among other things, Fig. 6 also reveals that regions of almost quiescent flow are created as a consequence of the decrease in the number of couple of rolls occurring when $\theta$ is increased. These regions correspond in the figure to the areas with less dense distribution of streamlines.

Taken together, all these figures indicate that, in general, an increase in $\theta$ causes an expansion in the horizontal extension of the rolls (this leading to a shrinkage of their number). The most interesting information emerging from a careful analysis of these modes of oscillatory convection (and the related "ranges of existence" summarised in Table V), however, is the realisation that, while stationary modes are no longer possible when $\theta$ exceeds the threshold 0.08 , coexistence of different solutions is still possible. Indeed overlap of oscillatory modes clearly occurs in the ranges $0.007 \leq \theta \leq 0.01,0.016 \leq \theta \leq 0.017$ and $017 \leq \theta \leq 0.021$ for the regimes [PBR(5), FRCE(5)], [HS(5), FRCE(4)] and [HS(4) and FRCE(4)], respectively. 
Moreover, on increasing the control parameter $\theta$ past 0.02 , a sub-region of "chaotic behaviour" is encountered just after the interval in which the FRCE(4) and HS(4) solutions coexist. Additional meaningful information about this special state can be gathered from Fig. 7, in which we have reported a sequence of typical phase portraits for different (increasing) values of $\theta$. These plots provide some evidence that for $\theta \cong 0.02$ random switching of the system trajectory among two or more concurrent attracting sets can occur. Indeed, the reader will easily realise that the evolution path shown in Fig. 7c looks like a trajectory formed by the reiterated superposition of those shown in Figs. 7a and 7b pertaining to FRCE(4) and HS(4) states, respectively.

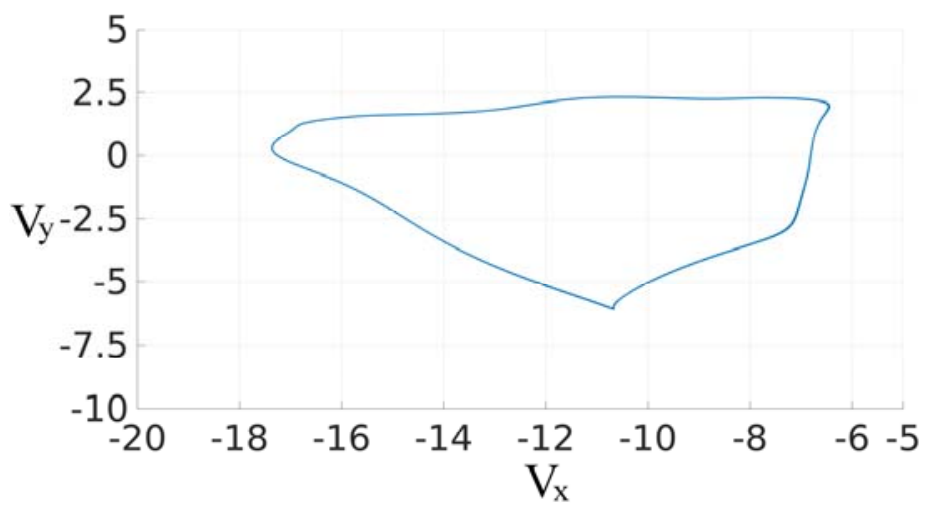

a)

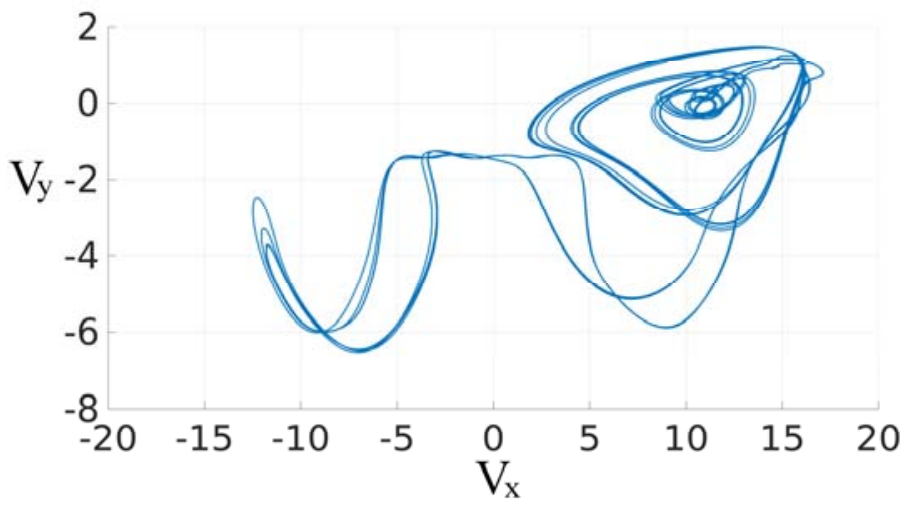

b)

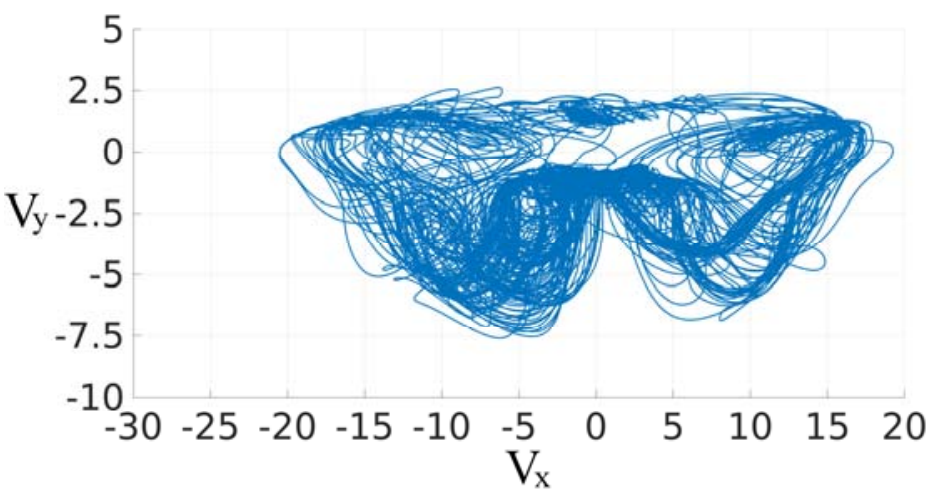

c)

Figure 7: Vertical components of velocity as a function of the horizontal components in a selected point of the physical space (phase portraits): a) $\theta=0.017$ (FRCE4), b) $\theta=0.019$ (HS4) c) $\theta=0.0218$ (chaotic flow). 
These observations confirm that the presence of multiple attractors can act as an additional source of randomness in a chaotic system producing sudden and repeated jumps from a branch of solutions to another branch.

Past $\theta=0.04$, however, the chaotic attractor reduces to a simple time-periodic flow following a chaos “crisis" similar to that observed in standard Rayleigh-Bénard convection, e.g., by Paul et al., ${ }^{58}$. As discussed, e.g., by Kitano et al., ${ }^{59}$, this kind of bifurcation can be viewed as a sudden change in the size of the attractor. In line with these studies and the general arguments provided by Grebogi et al., ${ }^{60}$ and Arnol'd et al., ${ }^{61}$, we argue that such intermittent behaviour is induced by the "collision" of the chaotic attractor with an unstable periodic orbit or its stable manifold. As the orbit approaches the unstable orbit its distance from the previous attractor increases, thereby driving the system towards a different scenario (represented by the PBR(3) branch in Fig. 3).
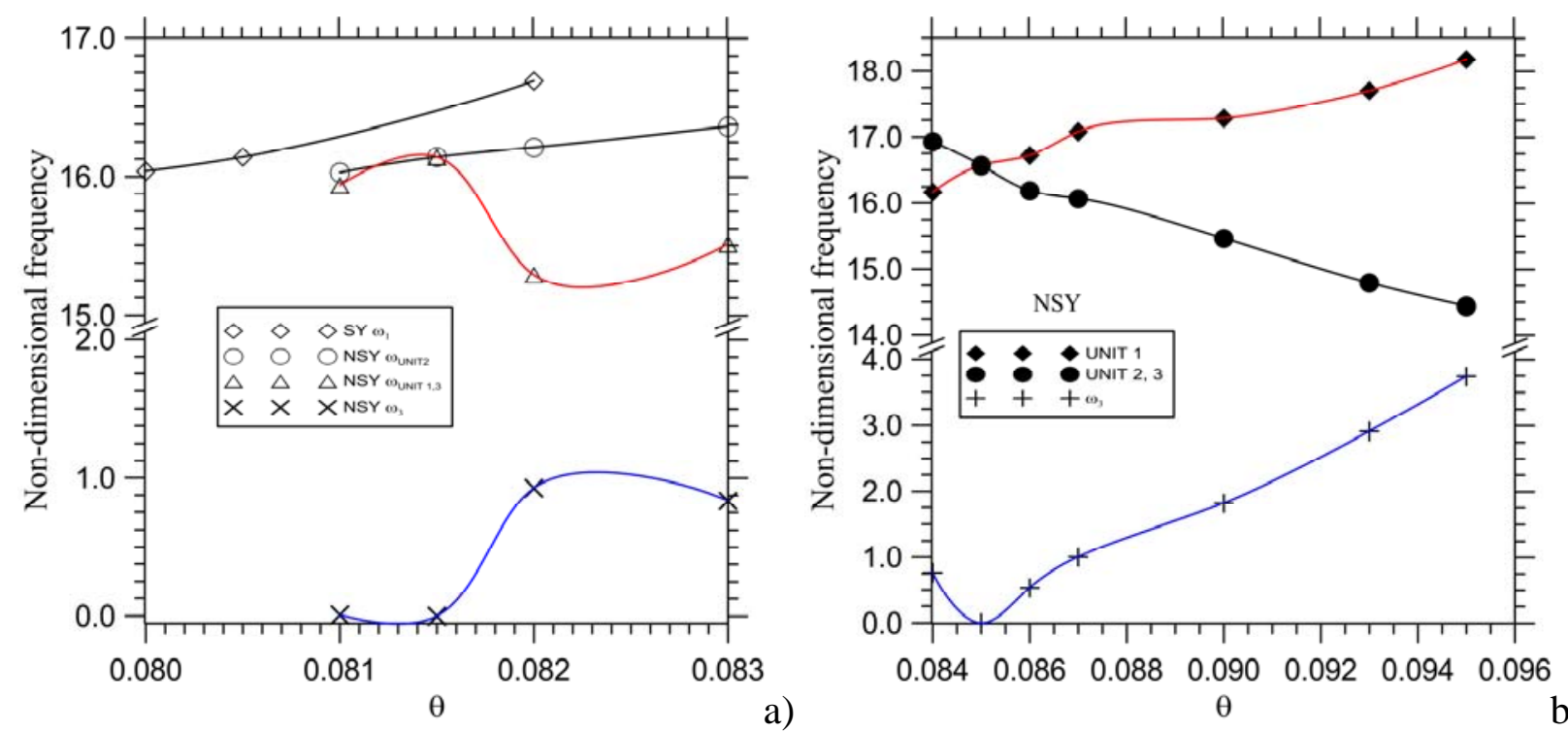

a)

b)

Figure 8: Multiple states of oscillatory convection [ a) $0.080 \leq \theta \leq 0.083$; b) $0.084 \leq \theta \leq 0.095$ ] categorized as SY regimes (where all the units oscillate in a synchronous fashion, each unit being formed by a roll and its counter-rotating companion - refer to the single black line) and NSY regimes (states with three units in which two units share the same frequency while the third displays a slightly different frequency - related frequencies being highlighted by the black and red interpolating lines, respectively). The additional blue line has been used to represent the long-period modulation produced for the NSY regimes by the non-linear interference between the other two oscillatory mechanisms. .

Continuing with the description of the new states revealed by the numerical simulations, it is worth pointing out that all these stationary and time-dependent solutions are taken over by a completely different scenario as $\theta$ exceeds another threshold. This is shown, in Fig. 8.

For $\theta \geq 0.08$, in particular, a new mechanism of oscillatory convection becomes dominant. It consists of the coexistence of different "units". For simplicity, hereafter we will use the term "unit" to 
denote the couple formed by a roll and its counter-rotating companion such that fluid is transported towards the surface at the centre and towards the bottom at the periphery of the couple.

Different branches of solutions can yet be identified in the space of parameters. These can be categorized as states with three units in which two units share the same frequency while the third displays a slightly different frequency (reported in Fig. 8a as NSY states) and other regimes (“ordered patterns") in which all the units oscillate in a synchronous fashion (SY states). Interestingly for the NSY branch, a long-period modulation is also present with frequency $\omega_{3}$ equal to the difference of the two other frequencies (see, e.g., Fig. 9). This phenomenon is produced by the obvious non-linear interference between the other two oscillatory mechanisms; "beats" are often observed in nature between oscillatory phenomena with similar frequencies.

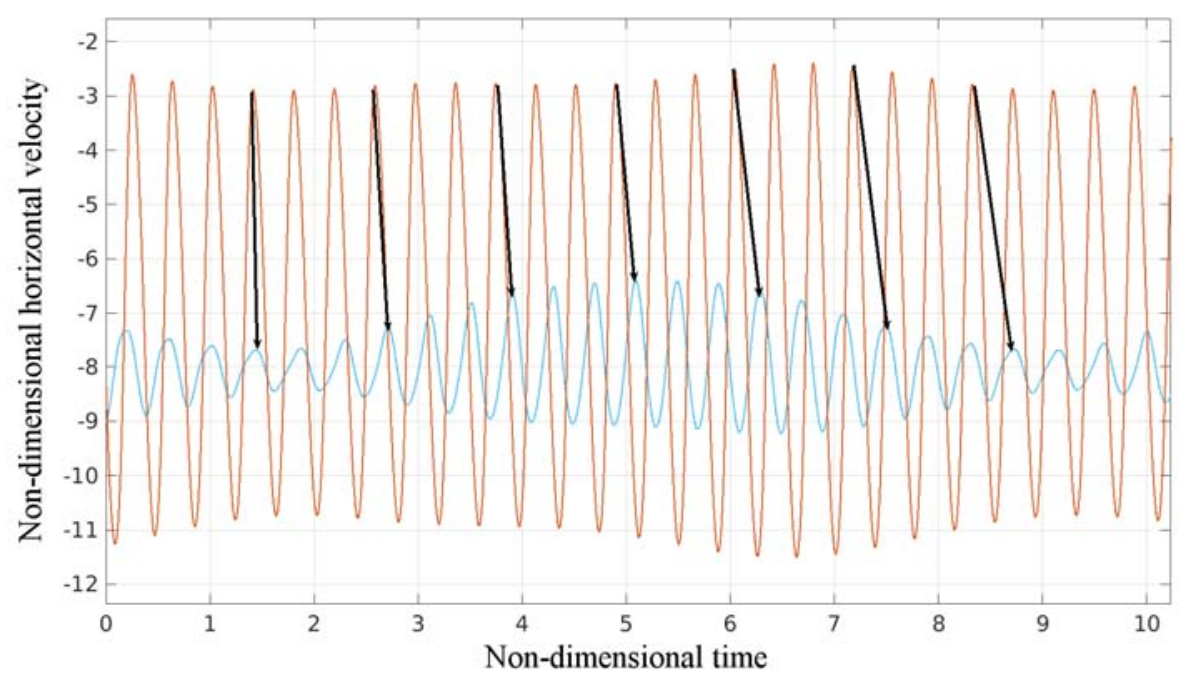

a)

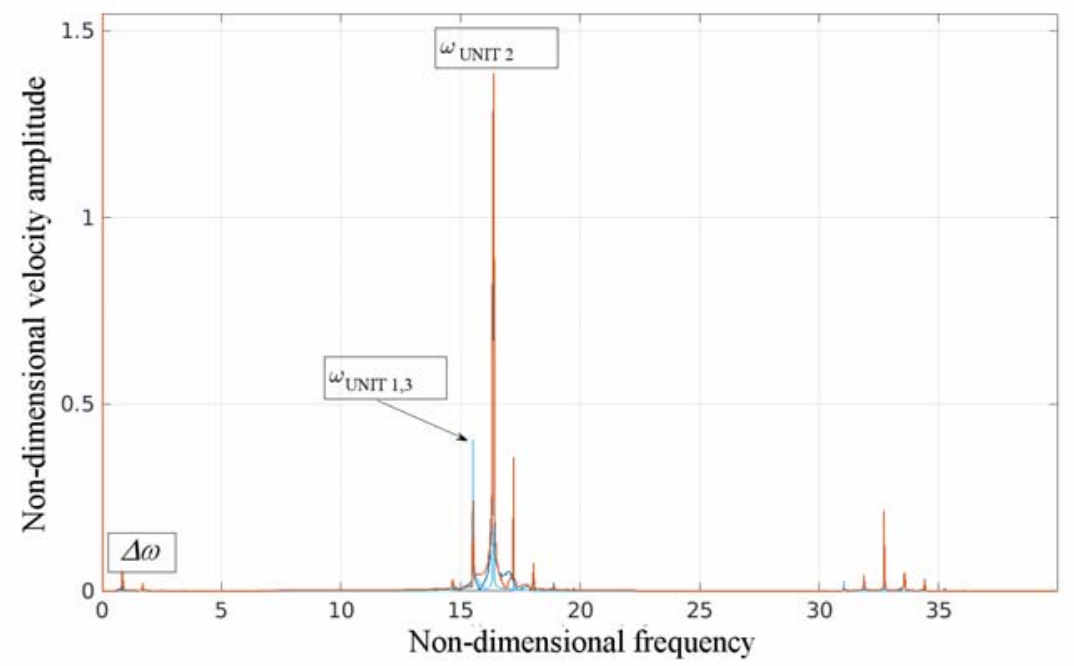

b)

Figure 9: Oscillatory convection for $\theta=0.083$ : a) time series; b) frequency spectrum (in blue the units 1 and 3, in brown the unit 2). 


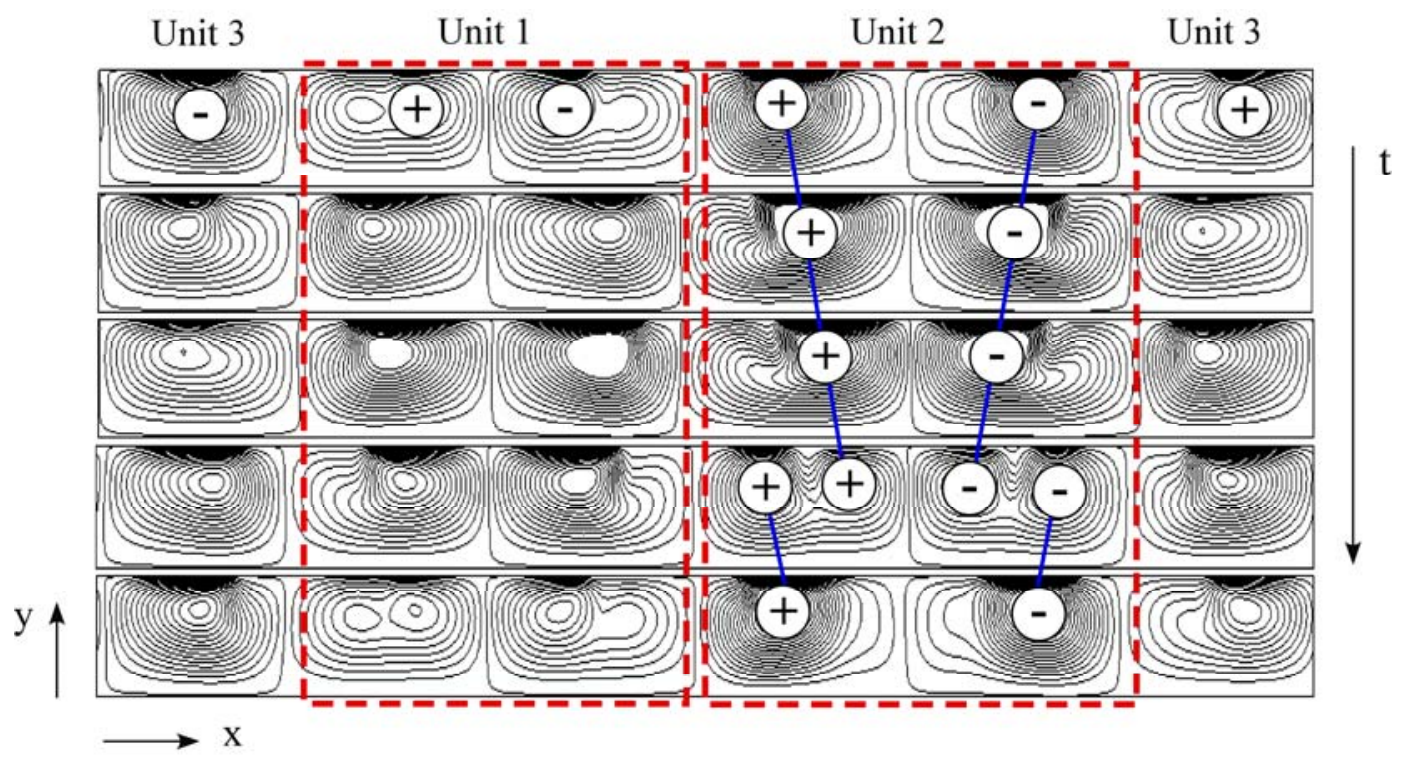

Figure 10: Sequence of snapshots evenly spaced in time revealing typical dynamics for $\theta=0.085$ (NSY) - The “+” and “-“ symbols indicate counter-clockwise and clockwise oriented rolls, respectively.

Most notably, however, if $\theta$ is further increased, the SY states featured by frequency locking, are no longer a solution. For $\theta \geq 0.084$ (Fig. 8b) only the NSY states survive (see, e.g., Fig. 10).

For $0.084 \leq \theta<0.088$, these attractors display essentially the typical behaviour of a $\mathrm{T}^{2}$ torus with two similar but incommensurate frequencies and related beats as shown in Fig. 8b. For $\theta \geq 0.088$, however, they clearly acquire a "fractal structure". This aspect is further illustrated in the following resorting to concepts and tools typical of the analysis of chaotic systems.

As an example, the peculiar properties of the rate with which a typical orbit "visits" different parts of the attractor can naturally be incorporated into the definition of the natural measure. This scalar quantity can represent the percentage of the time that a long orbit on the attractor spends in any given region of the state space.

A related concept is that of correlation dimension $\mathscr{D}_{2}$ (Balatoni and Renyi, ${ }^{62}$ ). This dimension is thought to be a measure of the number of "active modes" in the system, or of the "effective number of degrees of freedom" (Theiler ${ }^{63}$ ). Remarkably, this quantity can take non-integer values, which is generally regarded as a synonym of fractal behaviour or "fractalisation"; let us recall that a fractal was defined by Prof. Benoit B. Mandelbrot in 1975 as "a fragmented geometric shape that can be subdivided into parts, each of which is, at least approximately, a reduced-size copy of the whole", which implies the concept of non-integer dimension.

Algorithms able to yield an estimate of the correlation dimension have been originally developed by Grassberger and Procaccia ${ }^{64,65}$. The values taken by $\mathcal{D}_{2}$ for the conditions considered in the present work (computed with the algorithm by Grassberger and Procaccia ${ }^{64,65}$ ) when $\theta$ exceeds the value 0.084 have been collected in Fig. 11. By embodying the concept of progressively increasing structure on finer and finer length scales, the increasing values taken by the correlation dimension when $\theta$ becomes higher clearly reveal the emergence of features typical of chaos. 
This can be clearly seen in the range $8.6 \times 10^{-2} \leq \theta \leq 9.4 \times 10^{-2}$, where the correlation dimension takes non-integer values ranging between 2 and 3 (before it attains values $\cong 4$ for $\theta \cong 9.6 \times 10^{-2}$ ). Most remarkably, the behaviour of the system in this interval is even more interesting of what happens when (on further increasing $\theta$ ) $\mathscr{D}_{2}$ becomes larger than 3 .

The meaning of the above statement is not as straightforward as one would imagine as it has a hidden connection with another very important question in fluid-dynamics (a problem still of a very controversial nature), namely the number of degrees of freedom required to produce transition to turbulence (i.e. fractalisation).

According to the classical Ruelle-Takens scenario, a third independent frequency should appear, i.e. the attractor should become a hypertorus $\mathrm{T}^{3}$, before transitioning to a strange attractor.

In other words, according to this route at least two Hopf bifurcations would be required to produce chaos, the first causing the transition from a periodic regime to a quasi-periodic regime with two incommensurate frequencies, and the second leading to a new regime with three incommensurate frequencies. This is indeed formalised by the so-called Newhouse-Ruelle-Takens theorem (Newhouse et al., ${ }^{66}$ ), which asserts that a torus $\mathrm{T}^{3}$, under the actions of some perturbations, degenerates to a "strange attractor", and therefore the existence of three frequencies (i.e. three degrees of freedom) should be regarded as a necessary and sufficient condition for the emergence of a chaotic regime.

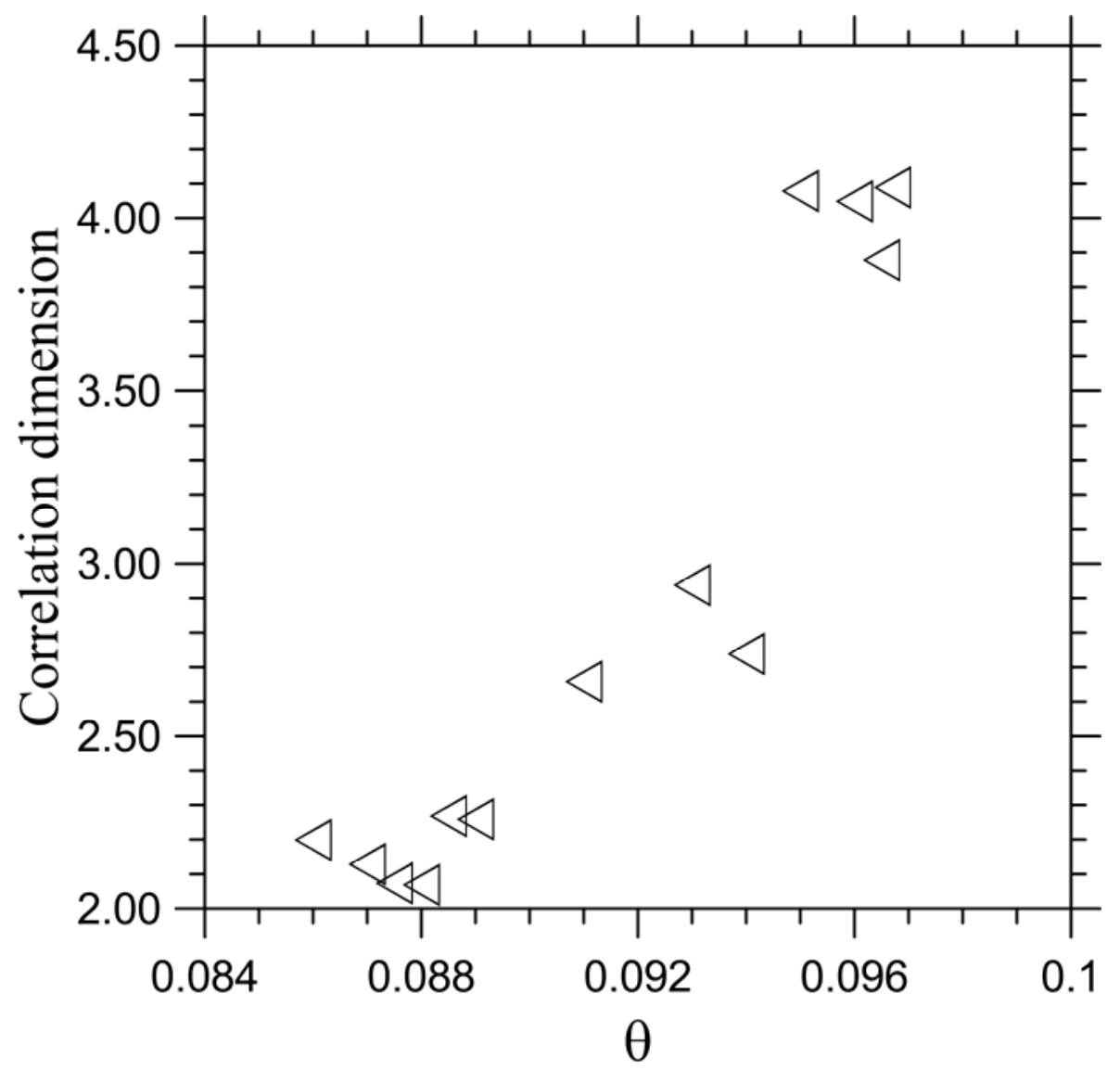

Figure 11: Correlation dimension as a function of $\theta$. 
On the one hand, this means that the present findings with fractalisation being produced starting from a $\mathrm{T}^{2}$ torus do support the alternate route towards chaos proposed by Curry and Yorke ${ }^{67}$ where transition to chaos is permitted from a torus $\mathrm{T}^{2}$; on the other hand they lead to another very important question, generally connected with the dynamics of non-linear systems and related theories, namely the problem relating to the existence of "universality classes" in such dynamics. The reader is also referred to the companion arguments elaborated in Sect. 5.

Here, we wish to emphasize that the Curry-Yorke scenario has been already reported for other problems in which viscoelastic fluids are involved, relevant examples being the Taylor-Couette flow $^{44}$ and Rayleigh-Bénard convection ${ }^{45}$. The main difference here is represented by the apparently crucial role played in the present problem by the coexistence of multiple solutions and the localised oscillatory structures (oscillons). As explained in Sect. 4.1, the latter become a persistent characteristic of the dynamics at relatively high values of $\theta$.

While the former (multiple states of convection) are a well-known property of thermogravitational convection in laterally heated liquid metals ${ }^{19,20}$, as discussed in the introduction, oscillons have been previously observed essentially in vibrated systems ${ }^{21-24}$.

Both features can be found in viscoelastic Marangoni-Bénard convection. This is confirmed by the three-dimensional (3D) simulations performed to probe the system response in a region of the space of parameters where according to $2 \mathrm{D}$ computations the initial torus $\mathrm{T}^{2}$ is taken over by a strange attractor (featuring oscillons as a pervasive characteristic of the flow). To do justice of these interesting findings, the next section is entirely devoted to these 3D results.

\subsection{Three-dimensional spatio-temporal dynamics}

A further understanding of the problem is gained by considering the role of the third spatial direction. Prior to embarking into an exhaustive description of such results, for the sake of completeness and clarity, in the following first we provide some initial and fundamental information about the modification displayed by the flow in terms of three-dimensional spatial structure as the elasticity number is increased. This strategy will prove very useful later when we will discuss the general features of such states from a temporal point of view and focus on the emergence of chaos and related dynamics.

Along these lines, we start from the simple observation that increasing $\theta$ has a remarkable impact on the typical topology of MB flow.

As illustrated in Fig. 12a, for $\theta=0$ (Newtonian fluid) the pattern is very regular and includes many triangular cells. Remarkably, the lines bounding these cells organize themselves to form a very well-defined network resembling the typical architecture of reticular trusses. Many of these lines originate from some special knots which behave as the centres of closed polygonal multi-cell structures having the shape of a "flower", with each triangular cell representing a distinct "petal" of the flower ${ }^{15}$. Many of such structures can be recognised in Fig. 12a. Though partial overlap among these sub-patterns is possible, the above-mentioned special knots can be uniquely identified through 
the topological order $\mathrm{p}$ of the radial spokes which originate from them. As evident in Fig. 12a, this topological order ( $\mathrm{p}_{\max }=$ maximum number of departing spokes) is $\mathrm{p}_{\max }=6$, whereas for standard patterns with the classical hexagonal and square cells typically found in Newtonian high-Pr liquids, $\mathrm{p}_{\max }$ would take values $\mathrm{p}_{\max }=3$ and $\mathrm{p}_{\max }=4$ (see, e.g., Lappa ${ }^{68}$ ).

As anticipated, however, an increase in $\theta$ causes significant adjustments. As evident in Fig. 12b, for a relatively small value of the elasticity parameter such as $\theta=7 \times 10^{-3}$, the just-discussed regular organisation is no longer a well-defined and recognizable characteristic of the pattern. Though the average extension of the different cells along the horizontal direction does not change significantly, the number $\mathrm{p}$ associated with the different knots becomes appreciably smaller, varying randomly through the physical domain between 3 and 5 (Fig. 12b).

The related network may be seen as an evolution of the reticular trusses seen in Fig. 12a due to the emergence of localised topological "defects" which prevent the formation of the regular polygonal multi-triangular-cell textures in some regions of the physical domain. However, a further increase in $\theta$ can lead to much more significant modifications of the overall flow structure. This is witnessed by the results shown in Fig. 12c for $\theta=3 \times 10^{-2}$. Indeed, apart from the much more disordered appearance of the pattern, a remarkable alteration affects the extension of the cells, which following a trend similar to that revealed by the 2D simulations (see, e.g., Fig. 4-6), grow in size (Fig. 12c). Notably, such expansion in the horizontal plane occurs in conjunction with a decrease in the overall number of cells as made evident by the emergence of surface regions of almost quiescent fluid. These areas, where the (horizontal) surface velocity is close to zero (while the component of velocity perpendicular to the free surface takes a relatively small but non-negligible value in the bulk fluid located underneath), "separate” the different cells (see again Fig. 12c). This trend is yet in agreement with our earlier observations about 2D flow (see Fig. 6 and related discussion in Sect. 4.1).

The extension of these "buffer" regions of return flow keeps increasing as $\theta$ becomes higher (see Figs. $12 \mathrm{~d}$ and Fig. 12e for $\theta=5 \times 10^{-2}$ and $\theta=9 \times 10^{-2}$, respectively). The initial configuration, crowded with a relatively large number of side-by-side cells, which transport heat from the bottom of the layer towards its surface in their centre and fluid in the opposite direction along their boundaries, is taken over by a progressively more sparse distribution in which cells look like (as seen in the temperature distribution) separated “islands” (the number of cells being depleted as $\theta$ increases, Fig. 13).

Having finished a description of the flow from a spatial perspective, we now turn to interpreting the related temporal dynamics. The progression from standard (Newtonian) Marangoni-Bénard convection to chaos as the elasticity number becomes higher can be appreciated by taking a look at Figs. 14-18. These figures reveal how, on increasing the elasticity parameter, (besides the aforementioned topological evolution) the pattern also undergoes substantial behavioural changes. 

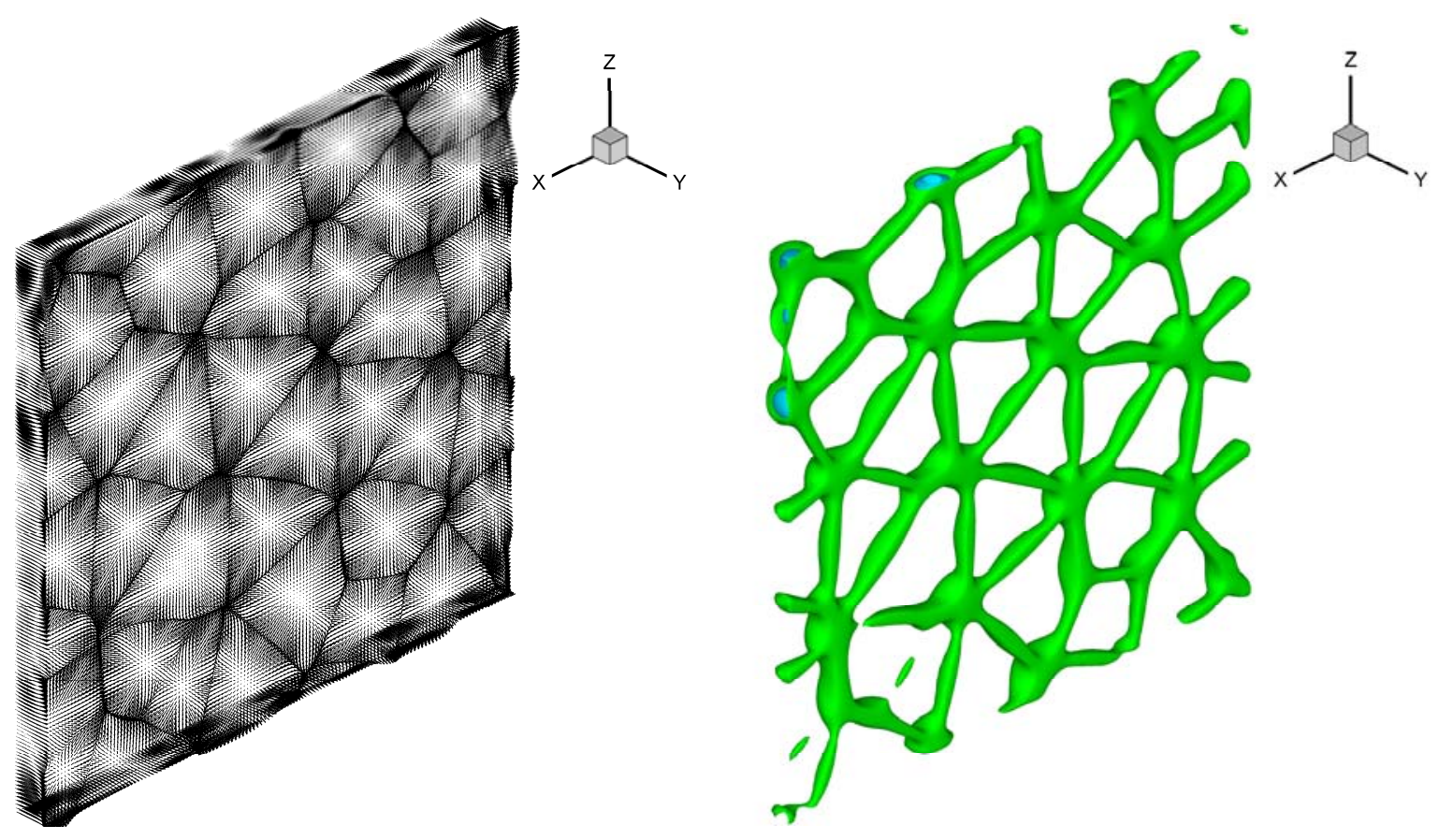

a)

Fig. 12 (see Pag. 24 for figure caption)
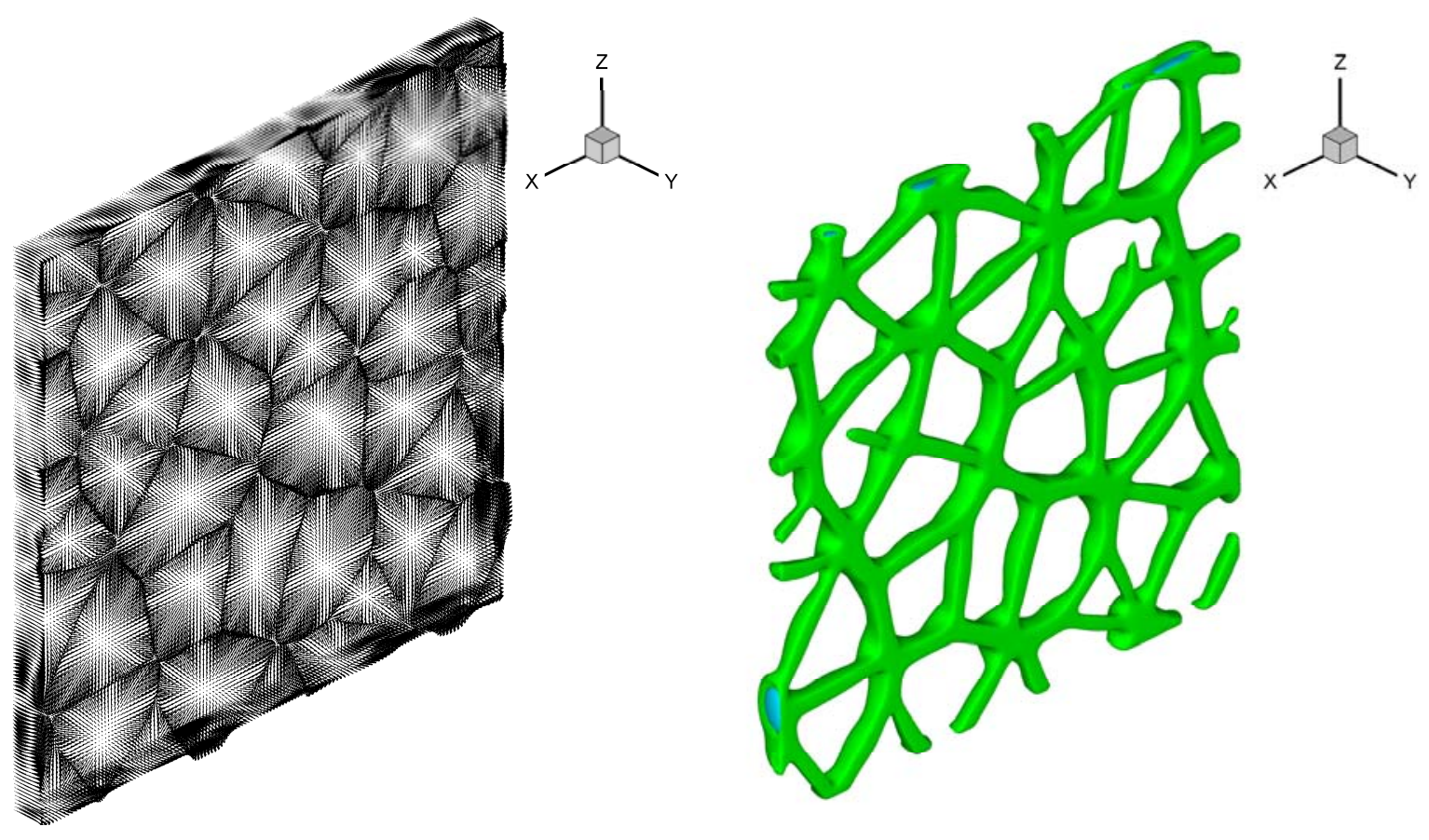

b) 

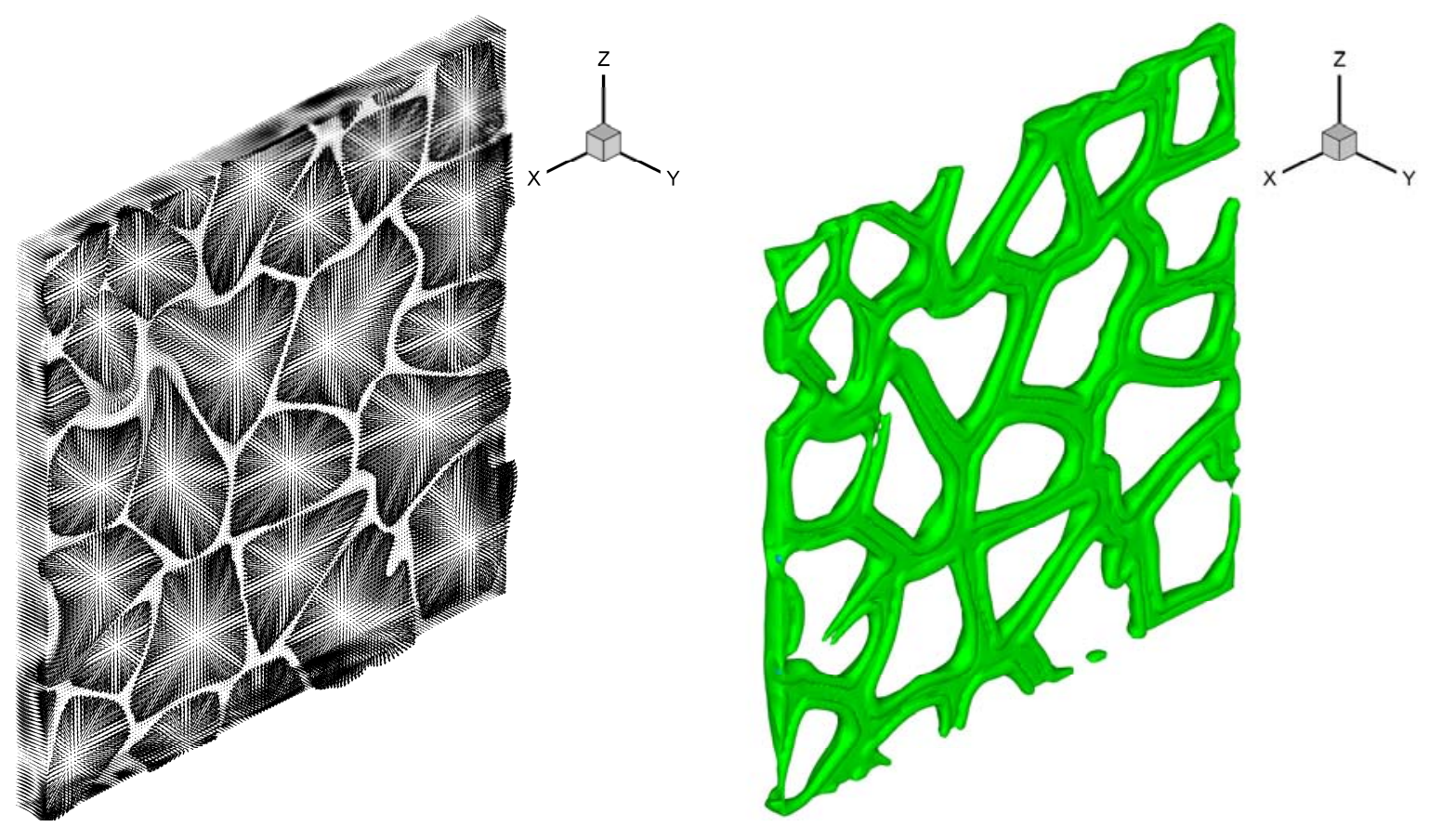

c)

Fig. 12 (cont'd)
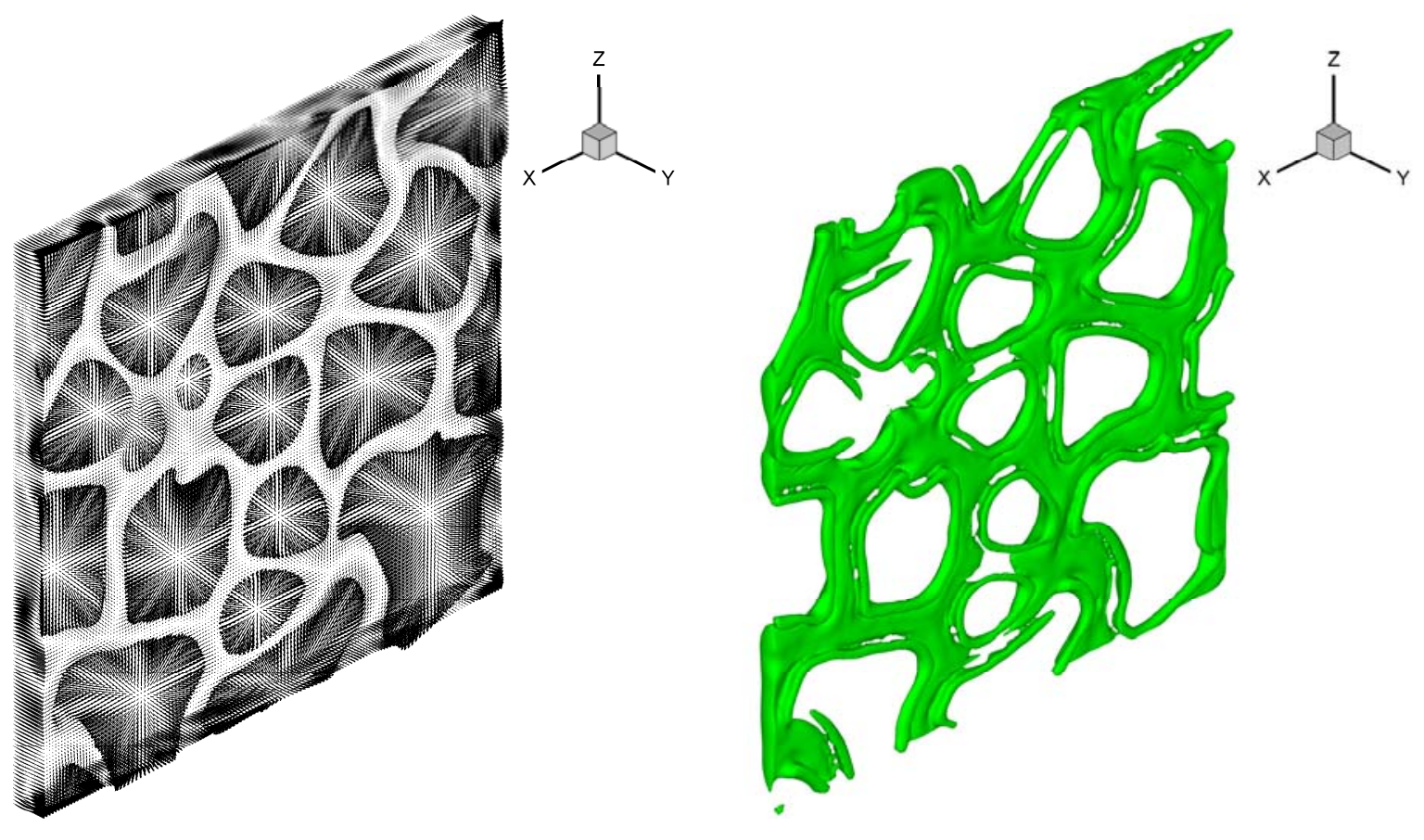

d) 

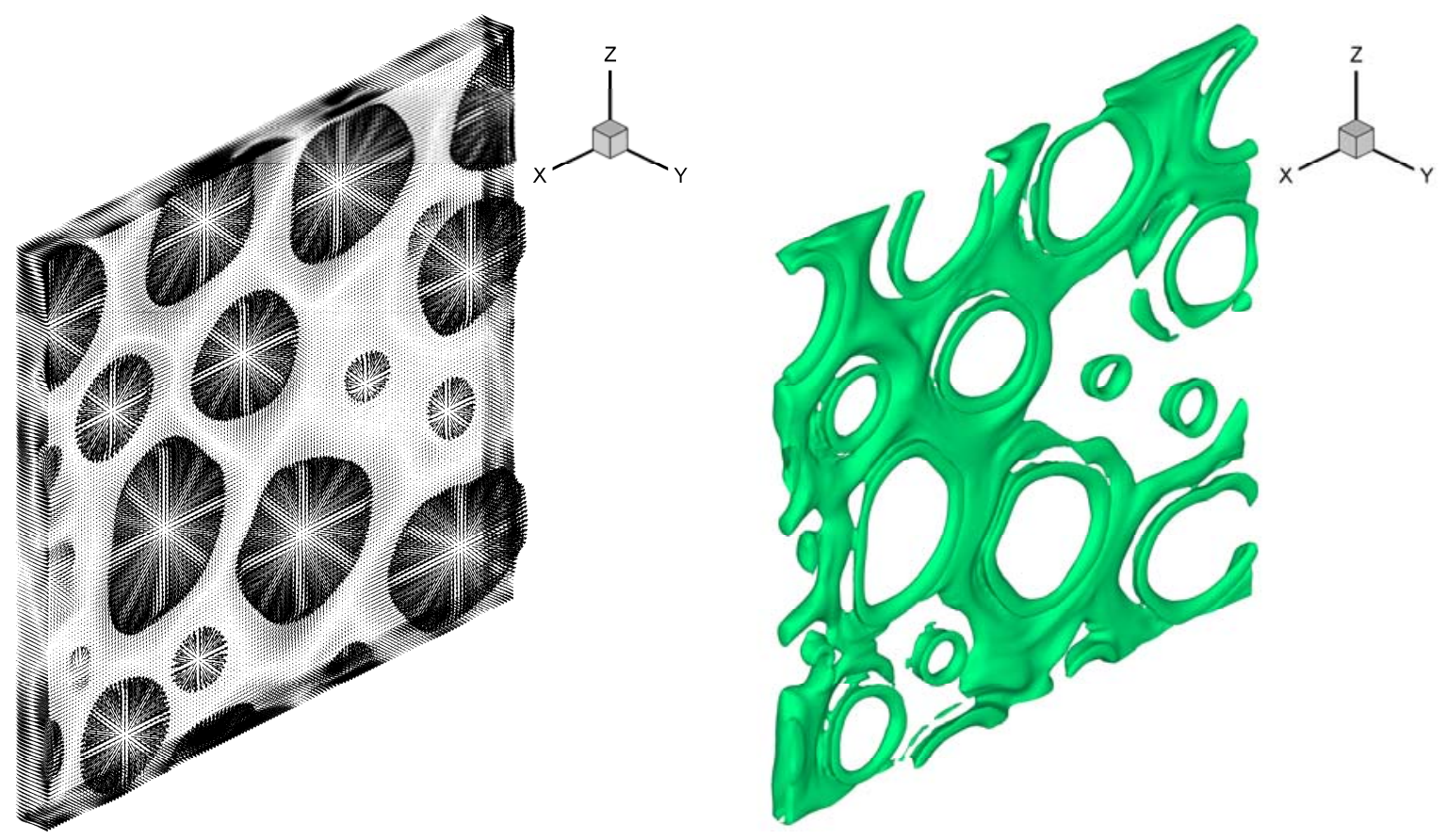

e)

Figure 12: $3 \mathrm{D}$ convection for $\mathrm{Ma}=300$ (snapshot of the vector field and related isosurfaces of the component of velocity perpendicular to the free surface, $15 \times 15 \times 1$ domain): a) $\theta=0$, b) $\theta=7 \times 10^{-3}$, c) $\theta=3 \times 10^{-2}$, d) $\theta=5 \times 10^{-2}$, e) $\theta=9 \times 10^{-2}$.

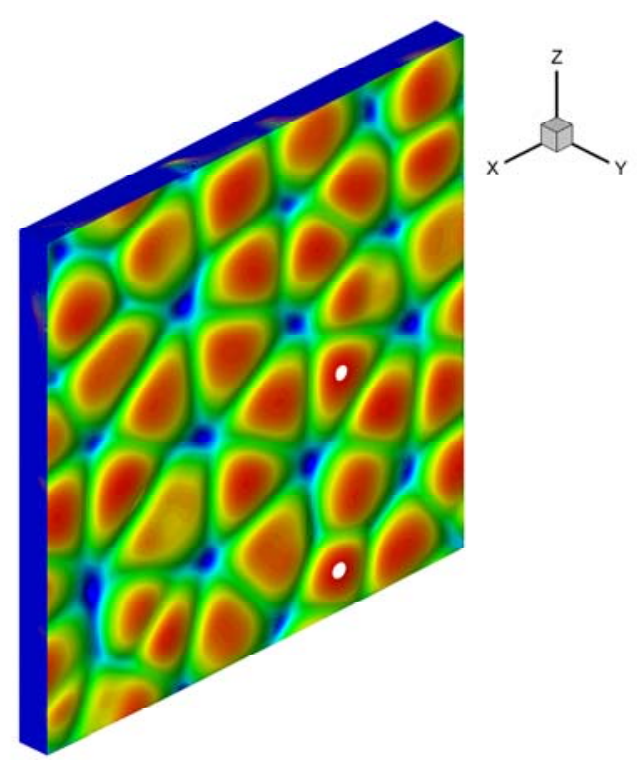

a) 


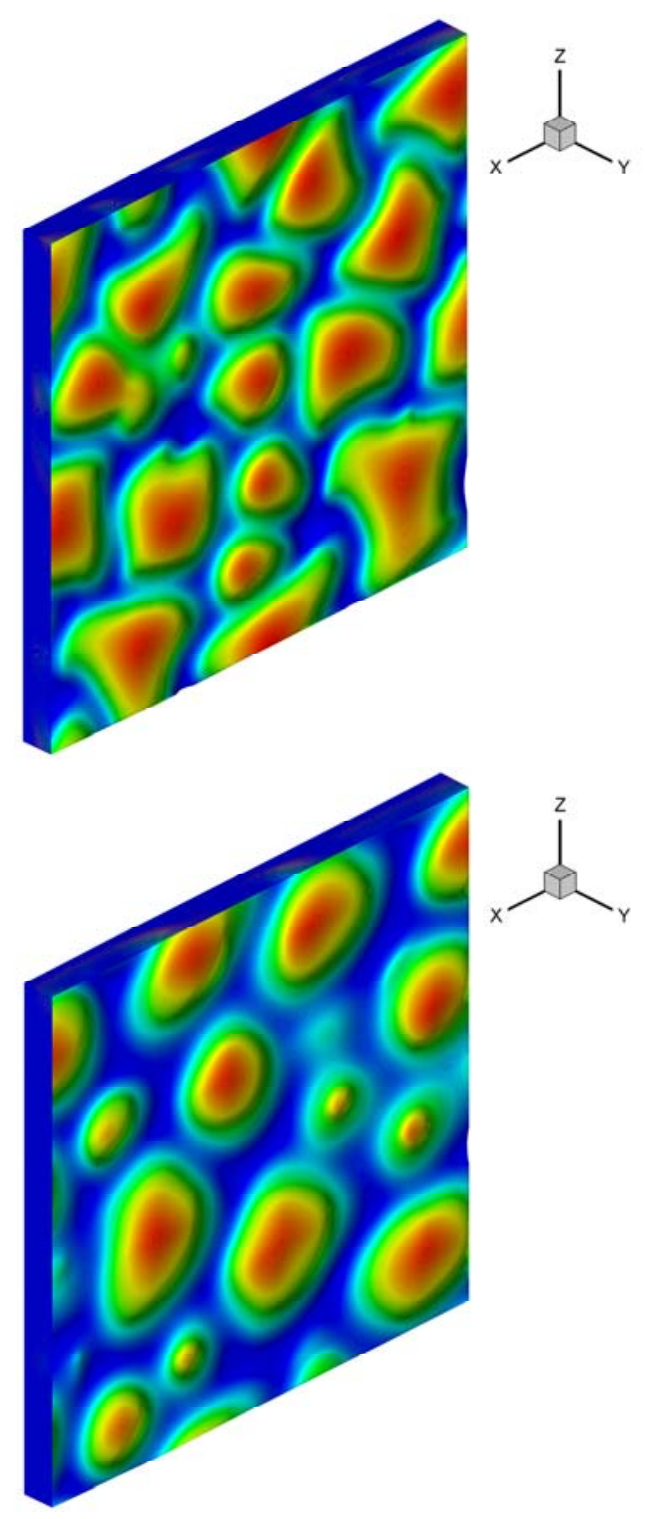

b)

c)

\section{T: $0.000 .090 .180 .27 \quad 0.36 \quad 0.450 .550 .640 .730 .820 .911 .00$}

Figure 13: Sequence of isosurfaces of temperature (snapshots) for different values of the elasticity parameter showing the island-formation effect: a) $\theta=0$; b) $\theta=5 \times 10^{-2}$; c) $\theta=9 \times 10^{-2}$.

When Newtonian conditions are established, the flow dependence on time is relatively weak. As shown in Fig. 14, the pattern retains a large-scale nearly steady structure in which the location and spacing of the reticular trusses with triangular topology is surprisingly regular ${ }^{15}$. For $\theta=3 \times 10^{-2}$, however, the irregularity visible in the spatial distribution of cells seen in Fig. 12c is also present in the temporal behaviour; this is confirmed by the temperature signals provided by a set of equally spaced thermocouples aligned along the $\mathrm{x}$ axis (Fig. 19a).

Comparison of Figs. 15 and 16 also leads to the very interesting realisation that the alterations are not limited to changes in the topology of the pattern and size of recognisable convective features. They also concern the morphology of the cells. Indeed, as $\theta$ is increased, such cells feature the development of cusps along initially straight sides, which are not possible at smaller $\theta$. 
Moreover, Fig. 16 reveals that since for $\theta=3 \times 10^{-2}$ the cells are no longer arranged in a tight side-byside configuration (as that seen in Fig. 14 or 15), they can continuously change shape (by developing, as mentioned before, new cusp points, i.e. by replacing straight sides with edges and vice versa).
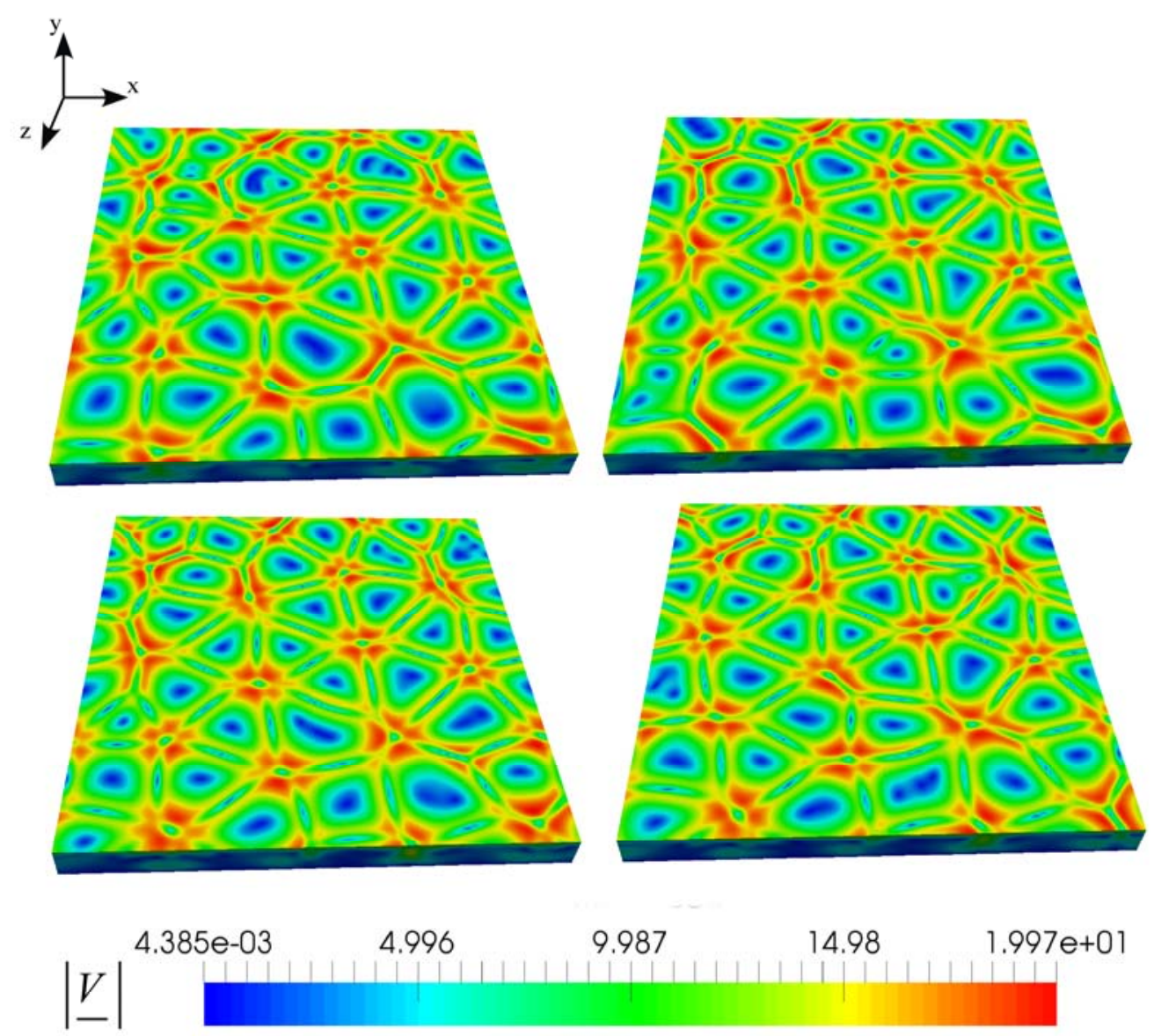

Figure 14: Snapshots of 3D convection (velocity module distribution at the free surface) evenly distributed in time for $\mathrm{Ma}=300$ and $\theta=0$ (15x15x1 domain). 

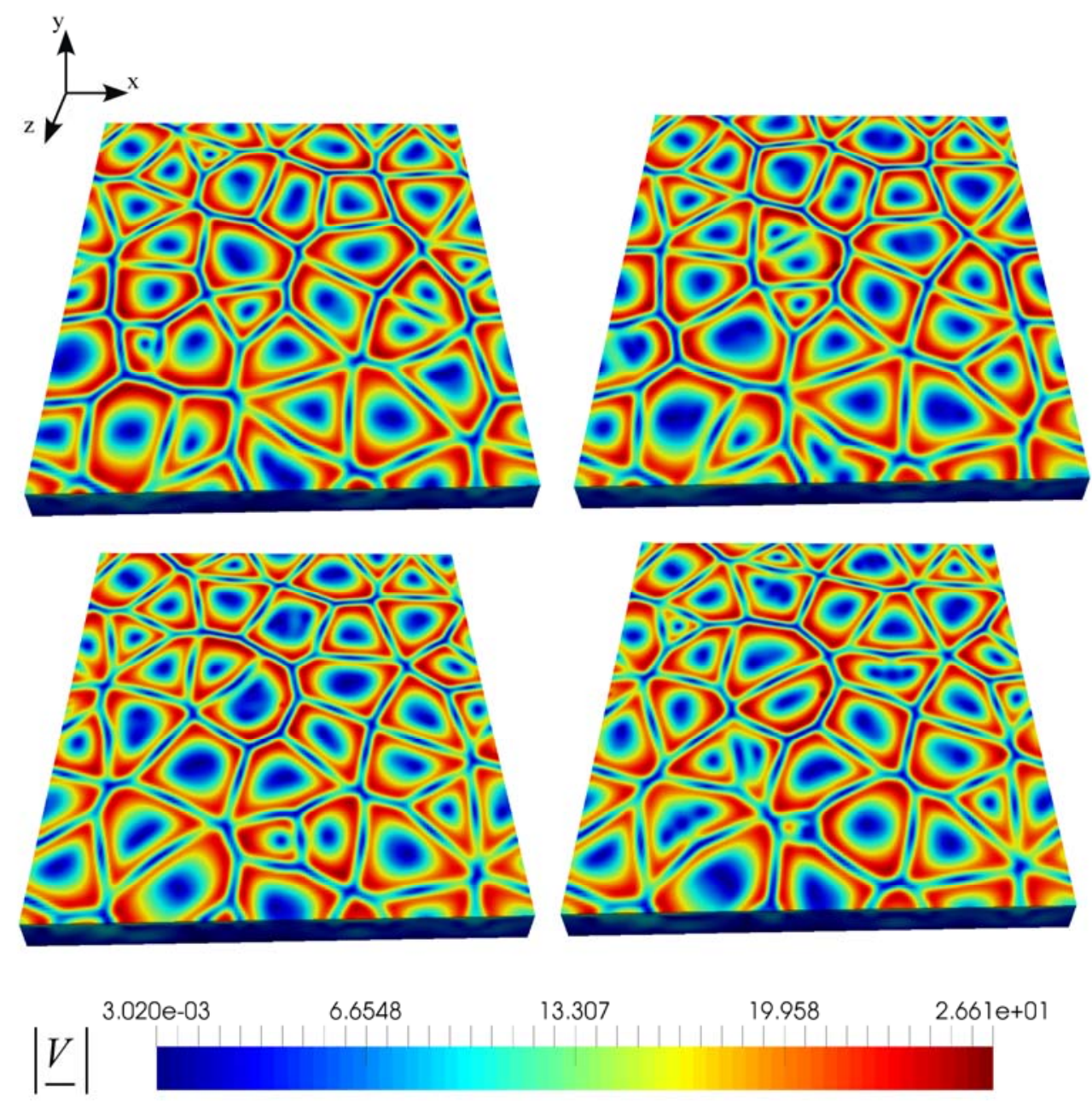

Figure 15: Snapshots of 3D convection (velocity module distribution at the free surface) evenly distributed in time for $\mathrm{Ma}=300$ and $\theta=7 \times 10^{-3}$ (15x15x1 domain).

This behaviour is retained and even reinforced for $\theta=5 \times 10^{-2}$ (Fig. 17) due to the increased space (buffer) being available between neighbouring cells. In addition, a new phenomenon starts to take place, namely the growth and decay of new (relatively small) cells nucleating in the buffer regions. Finally, as soon as $\theta$ exceeds a value similar to that already identified in the $2 \mathrm{D}$ case $(\cong 0.089)$ a chaotic state with apparently independent (well-separated in space) oscillons, which interact in time, is recovered (see Fig. 18 for $\theta=9 \times 10^{-2}$ ). For this case polygonal structures are no longer recognizable or evident. All the cells tend to acquire a more or less rounded boundary and change continuously shape as time progresses, their interactions resulting in an endless wandering process (supported or promoted by the increased spacing among them). Apart from the aforementioned nucleation phenomena occurring randomly in the physical domain, interestingly, in this state new 
dynamics are allowed in the form of cells that split (with a process closely resembling that of the cellular mitosis) or merge with other cells.

In such a context the additional information provided by equally spaced thermocouples aligned along a fixed direction (Fig. 19b) is still instructive as it clearly shows the "localised" nature and temporally limited duration of the events mentioned above (which might be regarded as "bursts" causing at certain positions and over finite-extent timeframes an increase in the frequency of oscillations).
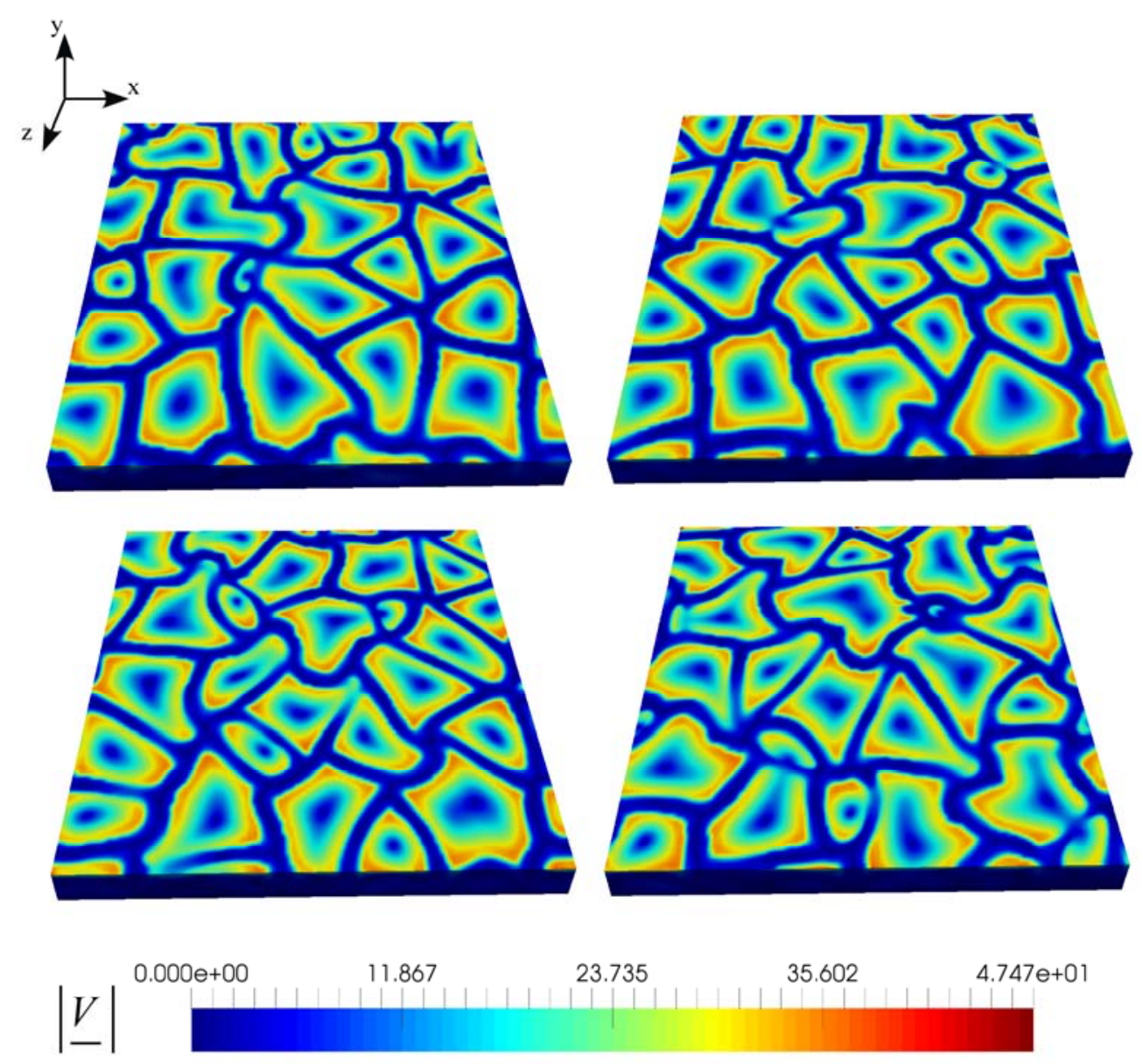

Figure 16: Snapshots of 3D convection (velocity module distribution at the free surface) evenly distributed in time for $\mathrm{Ma}=300$ and $\theta=3 \times 10^{-2}$ (15x15x1 domain). 


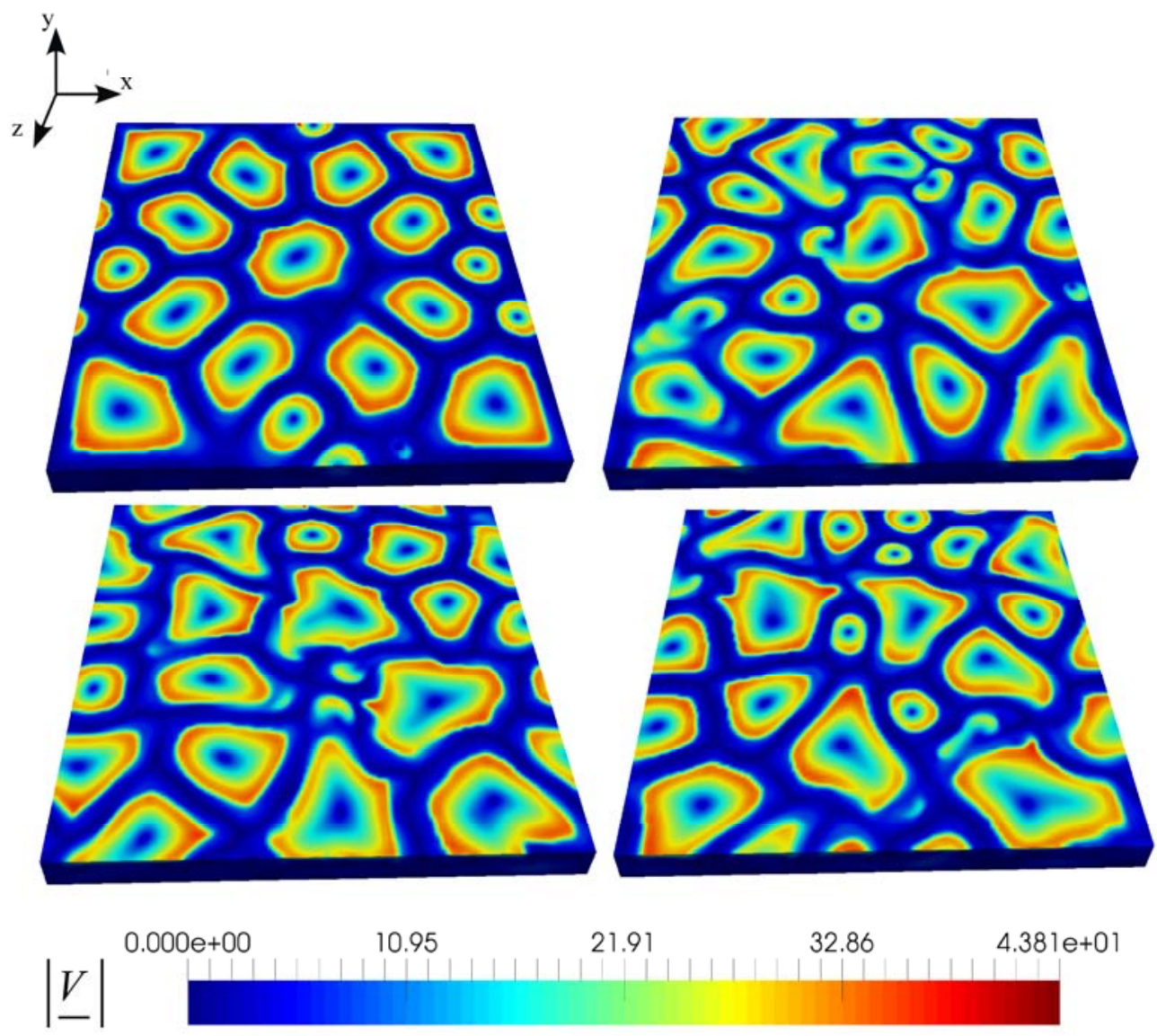

Figure 17: Snapshots of 3D convection (velocity module distribution at the free surface) evenly distributed in time for $\mathrm{Ma}=300$ and $\theta=5 \times 10^{-2}$ (15x15x1 domain).

Unfortunately, we could not track precisely the evolution of the system in terms of correlation dimension as the algorithm by Grassberger and Procaccia ${ }^{64,65}$ (and other similar approaches, see Theiler $^{63}$, Kostelich and Swinney ${ }^{69}$ ) works only for low-dimensional chaos, i.e. when the correlation dimension does not exceed 4; notably, similar concepts also apply to the Liapunov exponents and related quantities (see, e.g., Eckmann and Ruelle ${ }^{70}$ ). In line with the considerations reported in all these studies, our attempts to measure $\mathscr{D}_{2}$ for the above cases provided relatively high (not converging) values.

To conclude this section, we limit ourselves to mentioning that, most interestingly, this "ultimate" state of convection featuring convective cells that move, split or collide, like continents drifting in the ocean, displays some analogies with another kind of thermally driven chaotic flow, namely turbulent Rayleigh-Bénard (RB) convection in Newtonian fluids. Indeed, in turbulent RB, isolated thermal features in the form of plumes are known to emerge, grow and move chaotically in the physical domain, being subjected at the same time to 1) instabilities promoted by the thermal buoyancy itself or by the shear, which can cause new plumes to develop from the original plume stem (i.e. branching phenomena, see, e.g., Cortese and Balachandar ${ }^{71}$ ) or 2) clustering phenomena, which produce large-scale features (see, e.g., Solomon and Gollub ${ }^{72}$; Siggia ${ }^{73}$; Lappa $^{74}$ ). As shown in Fig. 18 in our case the dynamics are formally similar: oscillons nucleating at different positions 
in the domain can split into different parts or coalesce forming larger cells while wandering in the horizontal direction. We will come back to this interesting concept in Sect. 5 where this statement will be placed in a more general theoretical context in the light of existing theories on the subject.
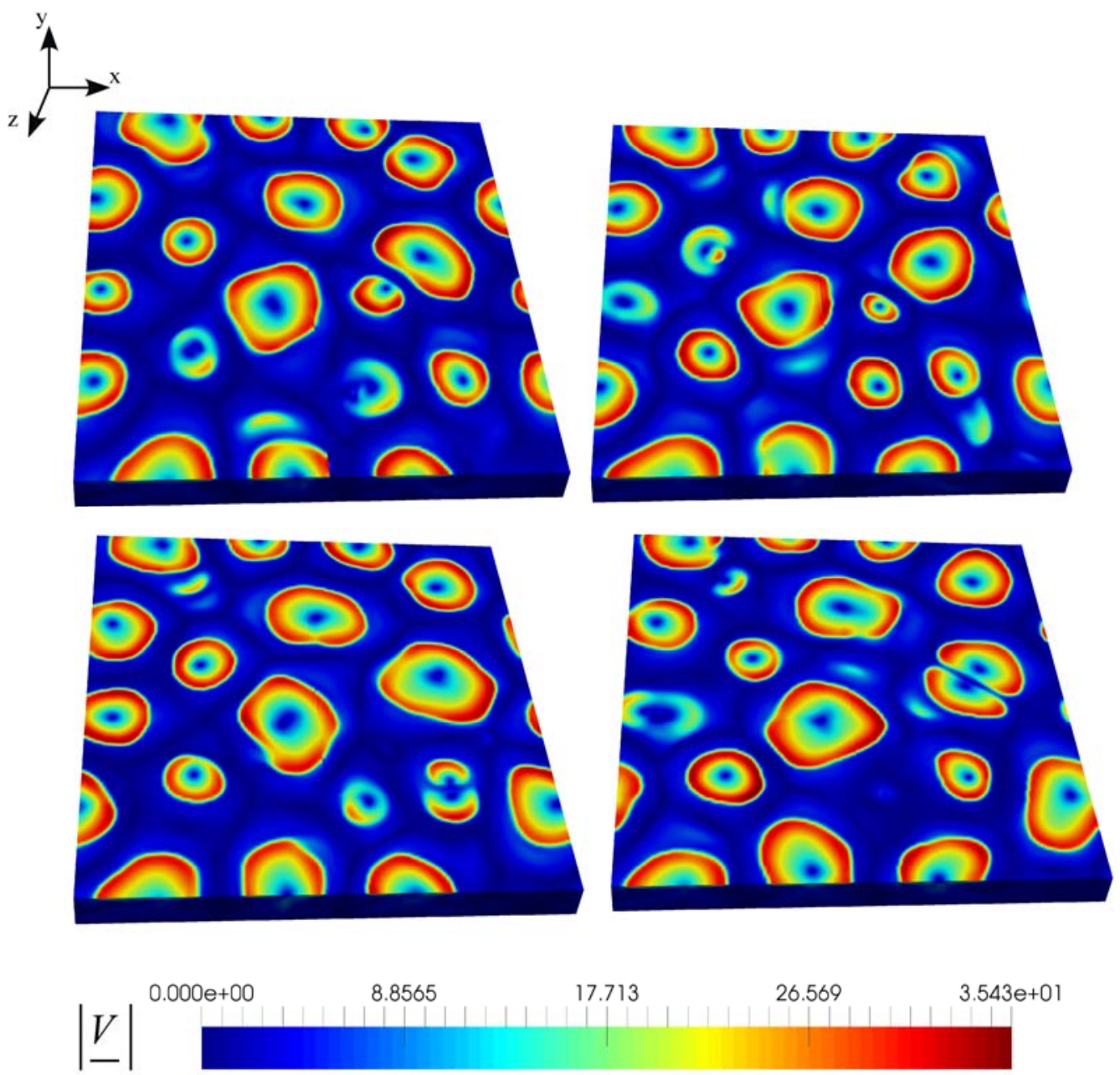

Figure 18: Snapshots of 3D convection (velocity module distribution at the free surface) evenly distributed in time for $\mathrm{Ma}=300$ and $\theta=9 \times 10^{-2}$ (15x15x1 domain). 

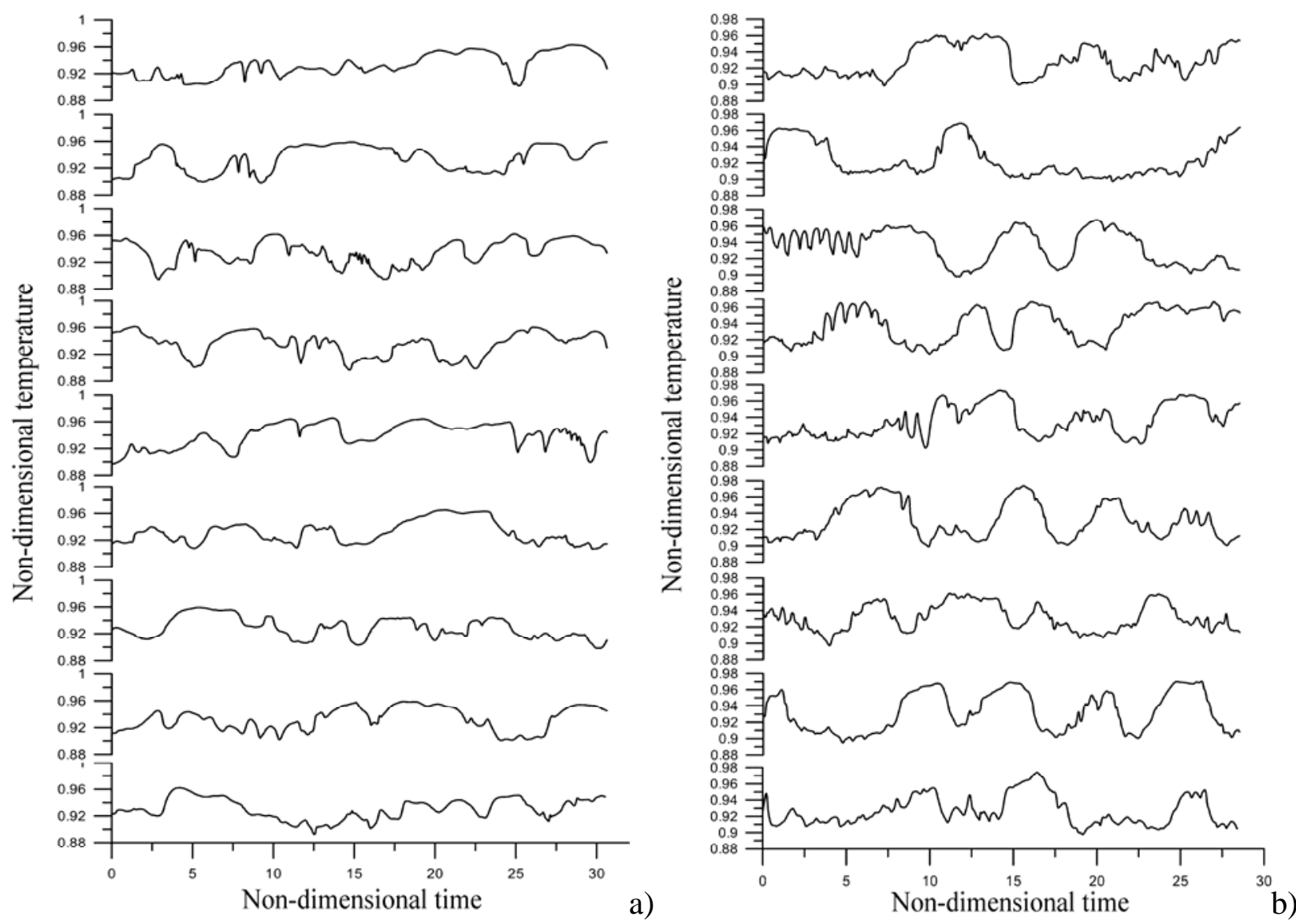

Figure 19: Signals provided by 9 thermocouples evenly spaced along the $\mathrm{x}$ direction $(\mathrm{Ma}=300$ and 15x15×1 domain): a) $\theta=3 \times 10^{-2}$, b) $\theta=9 \times 10^{-2}$.

\section{Discussion}

Towards the end to place the present findings in a more general context and get additional insights, there are some categories of flows and fluids that could be considered to establish interesting analogies (thereby enriching the discussion with some interdisciplinary flavour).

As already outlined in the introduction, oscillons are not an exclusive prerogative of the flow considered in the present work. Oscillons have been also observed in other contexts (e.g., granular media).

\section{$\underline{5.1 \text { Oscillons in granular media }}$}

Though granular media could be seen as a special kind of fluids, however, there are some special aspects that make their properties somehow unique, these being the dissipative nature of the interactions between grains (due to static friction) and the related collision phenomena, which contribute to the exchange of momentum at small scales (Tsimring and Aranson, ${ }^{23}$; Nikot, ${ }^{75}$ ). 
Though the connection between granular materials and viscoelastic fluids in terms of mechanisms responsible for exchange of momentum within the medium and related “emerging” properties (in terms of macroscopic pattern) at this stage is still unclear, some similarities can be highlighted. Indeed, recent experiments with vibrated granular materials have provided evidence for an astonishing variety of collective behaviours formally similar to those seen in the present work. These include (but are not limited to) standing waves, oscillatory patterns with hexagonal or square symmetry (also referred to as "granular waves"), one-dimensional worms (Jaeger et al., ${ }^{76}$ ) and oscillons (which emerge with finite initial amplitude in a hysteretic region between patterns and featureless states under the same conditions required for granular waves ${ }^{21,22}$ ).

Most interestingly, Umbanhowar et al., ${ }^{22}$ described how these localised features can interact. Several dynamics were reported, such as oscillons with the same phase displaying a short-range repulsive interaction, and oscillons of opposite phase attracting and "binding" to form stable dipole structures. Apart from dipoles, these authors also observed other stable configurations; among them, chains of oscillons (in which bound oscillons are able to influence each other even if they are not in direct contact) and triangular tetramers. Though structures of oscillons with square symmetry were not found, patterns with square symmetry (granular waves) were observed to oscillate with an arrangement similar to that of an "ionic crystal".

Similar dynamics have been reported for vibrated colloidal suspensions. Along these lines, Lioubashevski et al., ${ }^{24}$ clearly identified oscillons interacting and forming (depending on both the driving amplitude and initial conditions) localised patterns such as the triad structures of in-phase oscillons, short oscillon chains composed of either like- or unlike-phase oscillons, and patterns of oscillons with an internal hexagonal symmetry.

Though it is not easy to identify common cause-and-effect relationships with respect to the viscoelastic problem treated in the present work, perhaps some commonality could be seen in the ability of interacting oscillons to produce stable crystalline-like structures such as those that we have identified in a given range of $\theta$ (Fig. 8a). As shown in Fig. 8b, however, in the present case these single-frequency crystalline-like structures, which initially coexist with bimodal $\mathrm{T}^{2}$ states, are taken over as $\theta$ exceeds a given threshold by entities that display a fractal behaviour.

This places our problem in a more general context, namely, the transition to turbulence and related theories.

\subsection{Inertial and elastic turbulence and universality classes}

Perhaps the best way to address this specific point would be to start from an analysis of the general (known) characteristics of elastic turbulence and its differences with respect to standard inertial turbulence (see, e.g., Larson et al., ${ }^{77}$; $\operatorname{Larson}^{78}$; Groisman and Steinberg ${ }^{79}$; Morozov and van Saarloos ${ }^{80}$ ). A dichotomy is often drawn between these two forms of chaos.

Newtonian turbulence is, in general, ascribed to the role of inertial forces, which can destabilise the flow on increasing the Reynolds number. In turn, this implies that the velocity required for 
excitation of inertial turbulence must be somehow proportional to the viscosity of the considered Newtonian fluid. In other words, the more viscous is the considered fluid, the higher shall be its large-scale velocity. One may also state that this kind of turbulence is caused by excessive kinetic energy in parts of a fluid flow overcoming the opposite mitigating effect played by the fluid's viscosity. For this case, following the Kolmogorov's hypothesis (Kraichnan ${ }^{81}$ ), the existence of an "inertial" wavenumber region can even be postulated, where the kinetic energy is transferred from large scales towards small scales (the energy being partitioned among eddies in proportion to $\mathrm{k}^{-5 / 3}$, where $\mathrm{k}$ is the wavenumber, until it is dissipated at scales of the order of the so-called Kolmogorov length).

Though the above concepts have been found to work well in many circumstances in the case of Newtonian fluids (even for typical problems of thermal convection, see, e.g., Lappa and Gradisncak $^{13}$ and references therein), they are no longer applicable when elastic turbulence is considered.

Small-Reynolds number elastic turbulence arises when a certain value of the elasticity parameter is exceeded. Because (as illustrated for instance in Sect. 2.2 for the Oldroyd-B model), the polymer relaxation time, $\lambda$, usually grows proportionally to the polymer viscosity, this means, that, in principle, turbulence can be excited at lower velocities by using more viscous polymer solutions; this completely rules out the influence of inertia, which also explains why this kind of turbulence is often referred to as turbulence without inertia (Groisman and Steinberg ${ }^{82}$ ).

Even though an elaboration of a physical interpretation for the mechanism leading to elastic turbulence is not as straightforward as one would imagine, some general insights into its cause-andeffect relationships can be obtained on the basis of the mathematical arguments provided in Sects. 2.2 and 2.3 by observing that the most notable property of a polymer solutions is that the stresses are not immediately suppressed as the fluid velocity becomes zero (but rather decay with some characteristic time). This implies that the behaviour of a polymeric liquid has to depend on the history of the polymer molecules deformations, which provides these systems with a non-linear feedback mechanism potentially able to produce and amplify disturbances. The degree of nonlinearity in these mechanical properties is typically expressed via the so-called Weissenberg number $\mathrm{Wi}=\mathrm{V}_{\mathrm{r}} \lambda / \mathrm{L}$ where $\mathrm{V}_{\mathrm{r}}$ is a characteristic reference velocity for the considered problem. This number, which can be seen as a product of characteristic rate of deformation and the relaxation time, for the problem considered in the present work would read Wi=Ma $\theta$. By virtue of these feedback loops, effects relating to the stretching of polymer molecules can lead to new instability mechanisms; as an example, polymer molecules stretching caused by a primary flow, may cause irregular secondary flow able to exert their influence back on the polymer molecules, stretching them further and making the resulting flow increasingly turbulent, until a kind of saturated state is attained $^{79,82}$. It is in this way that nonlinearity can be regarded as "loop" that feeds information back into the system where it is iterated, or used multiplicatively; these arguments also apply the case of thermally driven viscoelastic flows, see, e.g., Khayat ${ }^{83,84}$. 
To some extent all these concepts apparently simplify the problem by abstracting some essential and distinctive features. Nevertheless, at this stage, we should expressly point out that, despite these remarkable differences between inertial and elastic turbulence, some notable (and unexpected) similarities can be identified between the present phenomena and another seemingly innocuous problem, namely the transition from laminar to turbulent (Newtonian) flow in an ordinary pipe.

This problem has been already considered in the past given its outstanding implications in the elaboration of a general theory for turbulence where the evolution towards chaos proceeds in form of localised patterns. Originating from the pioneering study of O. Reynolds in the late 19th century, it continues to burgeon and bring surprises to this day. That it is why it has been recently used as a workhorse to assess the role potentially played by the spatial coupling of transiently chaotic localised features in sustaining turbulence (Avila et al., ${ }^{85}$ ).

As originally illustrated by Wygnanski and Champagne ${ }^{86}$, turbulence develops in ordinary pipes in the form of localised patches generally known as "puffs". These localised features emerge as isolated islands (sub-regions with relatively limited spatial extension) in a laminar flow and are known to grow or decay according to the value taken by the control parameter (the Reynolds number for this specific situation, Eckhardt et al., ${ }^{87}$ ). From a temporal point of view, all these turbulent sites relax towards a laminar phase if the control parameter is below the critical point. However, as soon as such a threshold is exceeded, because of the spatial coupling among such sites which produces a statistical phase transition, there is always an appreciable fraction of chaotic sites that grows on increasing the Reynolds number.

The alternating laminar and turbulent regions typical of pipe flow are intrinsic to this problem and "place pipe flow in the larger theoretical framework of spatiotemporal intermittency" 85 , which somehow resembles the present dynamics (Figs. 3 and 11) in which the interaction among coexisting branches of solutions or oscillons can give rise to inlets of chaotic behaviour for moderate values of the elasticity parameter or "fractal" dynamics (in the sense that has been given to this adjective in the preceding pages) as this parameter exceeds a given threshold.

Such a similarity becomes even more interesting and meaningful if one takes into account that, as explained before, the physical mechanisms responsible for the emergence of high-Re turbulence in Newtonian fluids and low-Re chaos in viscoelastic fluids are, in general, thought to be very different.

Following up on the previous point, some additional information can be found in the aforementioned study by Avila et al., ${ }^{85}$, who correctly observed that, qualitatively, the behaviour with the spatial coupling of transiently chaotic domains giving rise to the sustainment of turbulence (such as that shown in Fig. 18), is reminiscent of the dynamics of a class of model systems called coupled map lattices $\left(\mathrm{Kanek}^{88}\right)$. This may, therefore, indicate the existence of a class of universality which transcends the differences between inertial and the elastic turbulence. Put simply, the above considerations might imply that non-linearities related to the specific way by which the considered medium transports or exchanges momentum at microscopic level may not be necessarily required to justify the observed behaviours, these being the manifestation of a general 
class of phenomena which is exclusive neither of granular media nor of viscoelastic fluids. Remarkably, this commonality may inspire in the future other fresh directions of interdisciplinary inquiry.

\subsection{Interacting localised convective features in other dissipative systems}

In order to stake out some common ground by providing a synthesis of other known relevant phenomena in Newtonian fluids where the coupling of spatially separated sites can give rise to the sustainment of turbulence, in the following we discuss other similar categories of phenomena. In particular we concentrate on known processes where the emergence of unsteady isolated features is somehow supported by the intrinsic or inherent tendency of the considered system to produce plumes, relevant examples being represented by binary fluid convection and moist turbulent convection.

As an example, Lappa ${ }^{89}$ investigated the pattern formation process driven by $\mathrm{N}$ droplets out of thermodynamic equilibrium, uniformly distributed (evenly spaced) on the bottom of a container filled with a partially miscible organic liquid (cyclohexane-methanol system), as a model of the typical phenomena which occur during the thermal processing of liquid-liquid systems exhibiting a miscibility gap (the so-called "immiscible alloys”). These special alloys exist as two immiscible liquid phases within a certain interval of temperature. In particular, for a monotectic system a homogeneous solution exists only at temperatures above the so-called critical temperature $\mathrm{T}_{c}$; if the temperature is decreased below $\mathrm{T}_{\mathrm{C}}$ the melt starts to decompose into two liquid phases of different composition and density which coexist, generally known as the "majority” and "minority" phases.

Initially these alloys behave as a homogeneous phase. However, as soon as the temperature is decreased by cooling the container from below, new droplets nucleate in the bulk of the liquid and move (due to sedimentation) to the colder bottom of the container where they start to dissolve because they are no longer in equilibrium with the surrounding phase. As a result, convention driven by solute gradients is produced by buoyancy effects around each droplet in the form of unsteady rising jets or plumes periodically released from the top of each droplet.

Remarkably, spatial coupling among these convective structures can be produced by the return flow associated with the primary plume generated by each droplet. As a result, the rising currents originating from the different droplets interact in unexpected ways giving rise to "ensemble behaviours", which may be regarded as another occurrence of dynamics produced by the mutual interference of oscillons.

Interestingly, Lappa ${ }^{89}$ observed the plumes (originating from different droplets) to produce in the initial stages of evolution "crystalline states" similar to those observed for the case of vibrated granular matter, with the frequency of plume emission being the same for all droplets and couples of droplets releasing plumes with opposite phase behaving as “dipoles”. However, progression from order to disorder was reported for later time, with the system undergoing transition to a condition in 
which the time-dependent release of solutal plumes becomes completely erratic from both spatial and temporal points of view ${ }^{90}$.

The route to chaos described in Sect. 4 also closely resembles that reported by Paul et al., ${ }^{58}$ for standard Rayleigh-Bénard convection in water. On increasing the control parameter, indeed, these authors obtained time-periodic rolls, quasi-periodicity, phase-locked states and chaos. Periodic and quasi-periodic states were found to reappear after the first chaotic window and develop chaos again through an 'attractor-merging crisis', i.e. the merging of “coexisting attractors”.

Other examples can yet be found in the literature for binary fluids. For instance, for the case of convective structures that are spatially confined and surrounded by quiescent fluid, it is worth considering the study by Barten et al., ${ }^{91}$ where nonlinear, spatially localised structures of traveling convection rolls in horizontal layers of binary fluids heated from below were investigated as a function of Rayleigh number for different conditions (two Soret coupling strengths, i.e. separation ratios, in ethanol-water mixtures).

Other relevant analogies could be drawn with regard to another very interesting phenomenon in nature (still involving plumes), i.e. moist turbulent convection, thereby offering another potential chance for cross-fertilisation of different literatures. As an example, recently Pauluis and Schumacher ${ }^{92}$ have focused on one particularly important regime, that is, the conditionally unstable layer that occurs when the atmosphere is stable for unsaturated parcels, but unstable for saturated parcels. Though this problem is yet closely related to the classical Rayleigh-Bénard convection (see, e.g., Lappa ${ }^{74}$ and the rich list of reference therein), it involves phase transitions between the gaseous and liquid phase and also depends on the effect of latent heat release on the buoyancy of air parcels. As a result, although unsaturated parcels are stable with respect to small vertical shifts, saturated moist air parcels produce saturated convective plumes.

The case of moist turbulent convection may be regarded as another example in which the spatial coupling of isolated entities can produce transition to chaos, with the role of oscillons seen in our case being replaced by the onset of isolated thermal plumes and related multiplicity (which depends on the effective spatial extension of the considered system). These authors observed states in which convection is suppressed or only present as a recharge-discharge mechanism, with short intense bursts of convection separated by long quiescent intervals or statistically stationary convective regimes.

\section{Conclusions}

The present results may be summarised as follows. In assessing the changes produced by an increase in the control parameter (elasticity number) in a problem of viscoelastic flow, it has been found that a seemingly innocuous and general system such as Marangoni-Bénard convection can display very rich and fascinating bifurcation scenarios.

Due to the disorganized appearance of the three-dimensional solutions (initially resisting a deeper analysis), we have followed a deductive approach with systems of growing complexity being 
considered as the discussion progresses. More specifically, we have initiated our investigation from the simplified conditions in which the number of involved spatial dimensions is reduced to two, which proved instrumental in unravelling processes that are interwoven or overshadowed in 3D.

As revealed by these results, while for relatively small values of $\theta$ multiple solutions occur in the form of ordered stationary multicellular states differing in wavelength and/or standing waves that coexist in the space of parameters, an increase in $\theta$ makes regimes with "localised features" the preferred mode of convection.

Indeed, we could identify the following sequence of events taking place along the ideal path bringing the system from initial laminar conditions towards chaos: initially, localised phenomena manifest in the form of an isolated oscillator in an otherwise stationary background flow; as $\theta$ is increased, however, the number of such features increases leading them to "interact".

Such interplay finally results into two coexisting possible lines of evolution, namely, it can produce completely synchronous behaviours (i.e. 'crystalline’ patterns) or $\mathrm{T}^{2}$ tori and associated "beats” due to the interaction between the two coexisting oscillatory modes in the same interval of the elasticity parameter (i.e. yet in the form of multiple solutions).

Two-dimensional simulations also proved to be a good testbed to get a "quantitative" measure of the fractal nature of these attractors. Indeed, given the relatively low-dimensional nature of chaotic states emerging under the constraint of 2D flow, we could evaluate precisely the so-called correlation dimension. By embodying the concept of progressively increasing structure on finer and finer length scales, this quantity can be used to reveal the emergence of features typical of chaos.

In this way we could clearly discern that the scenario with $\mathrm{T}^{2}$ tori remains relatively stable until, by further increasing the elasticity parameter past a critical value $(\theta \geq 0.088)$, the torus corrugates leading ultimately to strange attractors.

As shown by the three-dimensional simulations, the ultimate scenario with localised convective features, each independently contributing to increase the degrees of freedom of the system is retained if the third spatial dimension is considered. Such simulations (relatively limited in number due to their extremely high computational cost) allowed us to pinpoint a variety of interesting spatio-temporal dynamics.

Indeed, starting from conditions corresponding to Newtonian flow for which the pattern can be sketched as a very regular texture consisting of triangular cells, which self-organise giving rise to structures resembling flowers with symmetrically located petals, on increasing $\theta$, a complex sequence of modifications is enabled. Initially $\left(\theta \leq \mathrm{O}\left(10^{-2}\right)\right)$, these alterations essentially manifest in the form of localised defects in the flow topology, which prevent the pattern from displaying a regular polygonal multi-cellular structure in some sub-regions of the physical domain. For larger $\theta$ the topological alterations are coupled with significant modification in the morphological structure of the cells. Indeed, following a trend similar to that revealed by the 2D simulations, the cells grow in size, such expansion in the horizontal direction running in parallel with a decrease in the overall number of convective features, as witnessed by the emergence of surface areas of apparently quiescent fluid "separating” the different cells. 
The extension of these buffer regions keeps increasing as $\theta$ becomes higher until, as soon as $\theta$ exceeds a value similar to that already identified in the $2 \mathrm{D}$ case $(\cong 0.088)$, a chaotic state is produced with apparently independent (separated in space) oscillons. For this case polygonal structures are no longer a feature of the flow. All the oscillons have rounded boundaries and change continuously shape as time passes. Their interactions result in an endless wandering process manifested by islands moving back and forth along different directions that are not fixed but change in time (the instantaneous direction of horizontal motion not being necessarily the same for contiguous cells). This ultimate state also displays remarkable phenomena of cell nucleation, splitting and coalescence, which, taken together, are somehow reminiscent of the so-called "plate tectonics theory" and related "continental drift" (a phenomenon well-known to geophysicists, driven by the interplay of thermal plumes in the mantle of our planet). This key observation led us to sift through existing studies with different foci in order to glean hints and draw inferences about what factors may facilitate the transition to chaos in spatially extended systems that are dissipative in nature.

The affinity with other categories of phenomena seems to indicate that for the present conditions the emergence of chaos is driven by a process formally similar to that seen in problems where turbulence is sustained by the spatial coupling of unsteady localised features (relevant examples being the transition from laminar to turbulent flow in an ordinary pipe or the buoyancy flow in the form of plumes being produced by spatially separated sources of buoyancy). These arguments may be considered as a clue or hint for the existence of a class of universality that transcends the differences in the microphysical behaviour of different systems such as Newtonian fluids, viscoelastic liquids and granular media.

This commonality, which over recent years has led to the initiation of specific work devoted to the investigation of these aspects, can be regarded as the spark at the root of the present study.

On the basis of all these findings and related considerations, we conclude that the emergence of oscillons in the form of multiple solutions, which progressively increase the number of degrees of freedom of a dissipative system, may be regarded as another of the mechanisms potentially used by Nature to produce "fractalisation" that is the basic of creating turbulence through the development of strange attractors.

\section{Acknowledgements}

We would like to thank Dr. M. Oliveira (University of Strathclyde) for some useful discussions.

\section{References}

[1] Bergè P., Pomeau Y., and Vidal C., (1984), "Order Within Chaos-Towards a Deterministic Approach to Turbulence", John Wiley, New York, 1984.

[2] Bontoux P., Roux B., Schiroky G. H., Markham B. L., Rosenberger F., (1986), Convection in the vertical midplane of a horizontal cylinder. Comparison of two-dimensional approximations with three-dimensional results, Int. J. Heat Mass Transfer, 29 (2), 227-240. 
[3] Imaishi N., Yasuhiro S., Akiyama Y., Yoda S., (2001), Numerical simulation of oscillatory Marangoni flow in half-zone liquid bridge of low Prandtl number fluid, J. Cryst. Growth, 230, 164171.

[4] Shevtsova V.M., Melnikov D.E. and Legros J.C., (2003), Multistability of oscillatory thermocapillary convection in a liquid bridge, Phys. Rev. E , 68 (6), 066311 (14 pages).

[5] Shevtsova V., Mialdun A., Mojahed M., (2005), A study of heat transfer in liquid bridges near onset of instability, J. Non-Equilibrium Thermodynam., 30(3), 261-281.

[6] Lappa M., (2004), Fluids, Materials and Microgravity: Numerical Techniques and Insights into the Physics, Elsevier Science (2004, Oxford, England).

[7] Lappa M., (2013), On the variety of particle accumulation structures under the effect of gjitters, J. Fluid Mech., 726, 160-195.

[8] Gaponenko Y. and Shevtsova V., (2012), Heat transfer through the interface and flow regimes in liquid bridge subjected to co- axial gas flow, Microgravity Sci. Technol. 24 (4), 297-306.

[9] Mialdun A., Ryzhkov I.I., Melnikov D.E., and Shevtsova V., (2008), Experimental Evidence of Thermal Vibrational Convection in a Nonuniformly Heated Fluid in a Reduced Gravity Environment, Phys. Rev. Lett., 101, 084501.

[10] Liu J. and Ahlers G., (1996), Spiral-Defect Chaos in Rayleigh-Bénard Convection with Small Prandtl Numbers, Phys. Rev. Lett. 77, 3126-3129.

[11] Ahlers G., Grossmann S., and Lohse D., (2009), Heat transfer and large scale dynamics in turbulent Rayleigh-Bénard convection, Rev. Mod. Phys. 81, 503-537.

[12] Chillà F. and Schumacher J., (2012), New perspectives in turbulent Rayleigh-Bénard convection, The European Physical Journal E ,35,58 (25 pages).

[13] Lappa M. and Gradiscak T., (2018), On the Oscillatory Modes of Compressible Thermal Convection in inclined differentially heated cavities, Int. J. of Heat and Mass Transfer, 121, 412436.

[14] Saghir Z., Ahadi A., Mohamad A., Srinivasan S., (2016), Water aluminum oxide nanofluid benchmark model, Int. J. Therm. Sci., 109, 148-158.

[15] Lappa M., (2009), Thermal Convection: Patterns, Evolution and Stability, John Wiley \& Sons, Ltd (2009, Chichester, England).

[16] Lappa M., (2012), Rotating Thermal Flows in Natural and Industrial Processes, John Wiley \& Sons, Ltd (2012, Chichester, England).

[17] Crespo del Arco E., Pulicani P. P. and Randriamampianina A., (1989), Complex multiple solutions and hysteresis cycles near the onset of oscillatory convection in a $\mathrm{Pr}=0$ liquid submitted to a horizontal temperature gradient, C. R. Acad. Sci. Paris 309, II, 1869-1876.

[18] Pulicani J. P., Del Arco E. C., Randriamampianina A., Bontoux P., and Peyret R., (1990), Spectral simulations of oscillatory convection at low Prandtl number. Int. J. Numer. Meth. Fluids, 10(5), 481-517.

[19] Gelfgat A.Yu., Bar-Yoseph P.Z. and Yarin A.L., (1999), Stability of Multiple Steady States of Convection in Laterally Heated Cavities, J. Fluid Mech., 388, 315-334.

[20] Lappa M. and Ferialdi H., (2017), On the Oscillatory Hydrodynamic Instability of Gravitational Thermal Flows of Liquid Metals in Variable Cross-section Containers, Phys. Fluids, 29(6), 064106 (19 pages).

[21] Clément E., Vanel L., Rajchenbach J., Duran J., (1996), Pattern formation in a vibrated granular layer, Physical Review E., 53 (3), 2972.

[22] Umbanhowar P.B., Melo F. and Swinney H.L., (1996), Localised excitations in a vertically vibrated granular layer, Nature, 382, 793-796. 
[23] Tsimring L. S. and Aranson I.S., (1997), Localised and Cellular Patterns in a Vibrated Granular Layer, Phys. Rev. Lett. 79(2), 213-216.

[24] Lioubashevski O., Hamiel Y., Agnon A., Reches Z. and Fineberg J., (1999), Oscillons and Propagating Solitary Waves in a Vertically Vibrated Colloidal Suspension, Phys. Rev. Lett, 83 (16), 3190-3193.

[25] Busse F.H. and Whitehead J.A., (1971), Instabilities of convection rolls in a high Prandtl number fluid, J. Fluid Mech., 47, 305-320.

[26] Dennin M., Ahlers G., Cannell D.S., (1996), Spatiotemporal Chaos in Electroconvection, Science, 272(5260), 388-390.

[27] Riecke H. and Granzow G.D., (1998), Localization of Waves without Bistability: Worms in Nematic Electroconvection, Phys. Rev. Lett. 81(2), 333-336.

[28] Rogers J.L., Pesch W., and Schatz M.F., (2003), Pattern formation in vertically oscillated convection, Nonlinearity, 16, C1-C10.

[29] Pearson, J.R.A., (1958), On convection cells induced by surface tension, J. Fluid Mech., 4, 489-500.

[30] Schwabe D., (2006), Marangoni instability in small circular containers under microgravity, Exp. Fluids, 40, 942-950.

[31] Colinet P., Legros J.C., Velarde M.G., (2001), Nonlinear Dynamics of Surface-Tension-Driven Instabilities, John Wiley, 2001

[32] Bestehorn M., (1996), Square patterns in Bénard-Marangoni convection, Phys. Rev. Lett., 76,46-49

[33] Thess A. and Bestehorn M., (1995), Planform selection in Bénard-Marangoni convection: 1 hexagons versus g hexagons, Phys. Rev. E, 52, 6358-6367.

[34] Parmentier P.M., Regnier V.C., and Lebon G., (1996), Nonlinear analysis of coupled gravitational and capillary thermoconvention in thin fluid layers, Phys. Rev. E 54, 411-423.

[35] Getachew D. and Rosenblat S., (1985), Thermocapillary instability of a viscoelastic liquid layer, Acta Mech. 85, 137-149.

[36] Lebon G. and Cloot A., (1988), An extended thermodynamic approach of non-Newtonian fluids and related results in Marangoni instability problem, J. Non-Newt. Fluid Mech., 28 61-76.

[37] Lebon G., Parmentier P., Teller O., Dauby P.C., (1994), Bénard-Marangoni instability in a viscoelastic Jeffreys' fluid layer, Rheol. Acta 33, 257-266.

[38] Dauby P.C., Parmentier P., Lebon G., Grmela M., (1993), Coupled buoyancy and thermocapillary convection in a viscoelastic Maxwell fluid, J. Phys. Cond. Matter 5, 4343-4352.

[39] Siddheshwar P.G., Sekhar G.N., Jayalatha G., (2011), Surface tension driven convection in viscoelastic liquids with thermorheological effect, International Communications in Heat and Mass Transfer, 38, 468-473.

[40] Ramkissoon H., Ramdath G., Comissiong D., and Rahaman K., (2006), On thermal instabilities in a viscoelastic fluid, Int. J. Non-Linear Mech. 41(1), 18-25.

[41] Hernández Hernández I.J. and Dávalos-Orozco L.A., (2015), Competition between stationary and oscillatory viscoelastic thermocapillary convection of a film coating a thick wall, International J. Therm. Sci., 89, , 164-173.

[42] Wilson S.R.D., (1995), Growth rates of the Marangoni instability in a layer of elastic fluid, Rheol. Acta, 34, 601-605.

[43] Parmentier P., Lebon G., Regnier V., (2000), Weakly nonlinear analysis of Bénard-Marangoni instability in viscoelastic fluids, J. Non-Newtonian Fluid Mech. 89, 63-95.

[44] Khayat R.E., (1997), Low-dimensional approach to nonlinear overstability of purely elastic Taylor vortex flow, Phys. Rev. Lett., 78, 4918. 
[45] Li Z. and Khayat R.E., (2005), Finite-amplitude Rayleigh-Bénard convection and pattern selection for viscoelastic fluids, J. Fluid Mech., 529, 221-251.

[46] Revuz, D. and Yor, M. (1994). Continuous martingales and Brownian motion. Grundlehren der Mathematischen Wissenschaften [Fundamental Principles of Mathematical Sciences] Volume 293 (Springer-Verlag, Berlin).

[47] Alves M.A., Oliveira P.J., Pinho F.T., (2003), Benchmark solutions for the flow of Oldroyd-B and PTT fluids in planar contractions, J. Non-Newtonian Fluid Mech., 110, 45-75.

[48] Bird R. B., Curtiss C.F., Armstrong R. C. and Hassager O., (1987), Dynamics of Polymeric Liquids, vol. 2, 2nd edn. John Wiley \& Sons, New York.

[49] Bonito A., Clément P., Picasso M., (2011), Viscoelastic Flows with Complex Free Surfaces: Numerical Analysis and Simulation, pp. 305-370 In Handbook of numerical analysis, Special Volume: Numerical Methods for Non-Newtonian Fluids, Guest Editors: R. Glowinski and J. Xu, Nort-Holland (Elsevier, 2011).

[50] Hu K.X., He M., and Chen Q.S., (2016), Instability of thermocapillary liquid layers for Oldroyd-B fluid, Phys. Fluids, 28, 033105.

[51] Dee G. T. and Sauer B. B., (1998), The surface tension of polymer liquids, Adv. Phys. 47(2), 161-205.

[52] Lappa M., (1997), "Strategies for parallelizing the three-dimensional Navier-Stokes equations on the Cray T3E”; Science and Supercomputing at CINECA, 11, 326-340. ISBN-10: 88-86037-031, M. Voli, Editor (Bologna, Italy).

[53] Jang D.S., Jetli R., Acharya S. (1986), Comparison of the PISO, SIMPLER and SIMPLEC algorithms for the treatment of the pressure-velocity coupling in steady flow problems, Numer. Heat Transfer 10, 209-228.

[54] Moukalled F., Mangani L. and Darwish M., (2016), The Finite Volume Method in Computational Fluid Dynamics - An Advanced Introduction with OpenFOAM and Matlab, Springer International Publishing, 2016, New York).

[55] Rhie C.M. and Chow W.L., (1983), Numerical study of the turbulent flow past an airfoil with trailing edge separation. AIAA J 21,1525-1532

[56] Favero J., Secchi A., Cardozo N., and Jasak H., (2009), Viscoelastic Fluid analysis in internal and in free surface flows using the software OpenFOAM, Computers \& Chemical Engineering, vol. 34, no. 12, pp. 1984 - 1993, 2010. 10th International Symposium on Process Systems Engineering, Salvador, Bahia, Brasil, 16-20 August 2009.

[57] Kengne J., Nguomkam Negou A., Tchiotsop D., Kamdoum Tamba V., Kom G.H., (2018), On the Dynamics of Chaotic Systems with Multiple Attractors: A Case Study. In: Kyamakya K., Mathis W., Stoop R., Chedjou J., Li Z. (eds) Recent Advances in Nonlinear Dynamics and Synchronization. Studies in Systems, Decision and Control, vol 109. Springer, Cham.

[58] Paul S., Wahi P., Verma M.K., (2011), Bifurcations and chaos in large-Prandtl number Rayleigh-Bénard convection, Int. J. Non-Linear Mech., 46, 772-781.

[59] Kitano K., Yabuzaki T. and Ogawa T., (1984), Symmetry-recovering crises of chaos in polarization-related optical bistability, Phys. Rev. A 29(3), 1288-1296.

[60] Grebogi C., Ott E. and Yorke J. A., (1983), Crises: Sudden Changes in Chaotic Attractors and Chaotic Transients, Physica D 7(1-3), 181-200.

[61] Arnol'd V.I., Afraimovich V.S., IlyashenkoYu.S. and Shilnikov L.P., (1993), Bifurcation theory and catastrophe theory. In Dynamical Systems, vol. 5, Berlin and New York: Springer.

[62] Balatoni J. and Renyi A., (1956), Publ. Math. Inst. Hung. Acad. Sci. 1, 9 (in Hungarian) [translation: Selected papers of A. Renyi, Vol. 1 (Budapest Academy, 1976) p. 558]. 
[63] Theiler J., (1986), Spurious dimension from correlation algorithms applied to limited timeseries data, Physical Review A, 34(3), 2427-2432.

[64] Grassberger P. and Procaccia I., (1983a), Characterization of Strange Attractors, Phys. Rev. Lett., 50, 346-349.

[65] Grassberger P. and Procaccia I., (1983b), Measuring the strangeness of strange attractors, Physica D, 9, 189-208.

[66] Newhouse S., Ruelle D. and Takens T., (1978), Occurrence of Strange Axiom-A Attractors

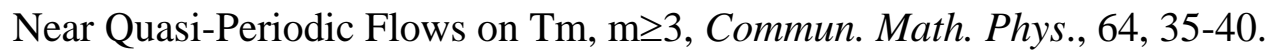

[67] Curry J and Yorke J.A., (1977), A transition from Hopf bifurcation to chaos: computer experiments with maps R2, in The structure of attractors in dynamical systems, Springer Notes in Mathematics, 668, 48.

[68] Lappa M., (2017), An alternative theoretical approach for the derivation of analytic and numerical solutions to thermal Marangoni flows, Int. J. of Heat and Mass Transfer, 114, 407-418.

[69] Kostelich E.J. and Swinney H.L., (1989), Practical Considerations in Estimating Dimension from Time Series Data, Physica Scripta, 40, 436-441.

[70] Eckmann J. -P. and Ruelle D., (1991), Fundamental Limitations for Estimating Dimension and Liapunov Exponents in Dynamical Systems, Physica D: Nonlinear Phenomena, 56(2-3), 1-4.

[71] Cortese T. and Balachandar S., (1993), Vortical nature of thermal plumes in turbulent convection, Phys. Fluids A, 5, 3226-3232.

[72] Solomon T.H. and Gollub J.P., (1990), Sheared boundary layers in turbulent Rayleigh-Bénard convection, Phys. Rev. Lett., 64, 2382-2385.

[73] Siggia E., (1994), High Rayleigh number convection, Annu. Rev. Fluid Mech., 26, 137-168.

[74] Lappa M., (2011), Some considerations about the symmetry and evolution of chaotic Rayleigh-Bénard convection: The flywheel mechanism and the "wind" of turbulence, Comptes Rendus Mécanique, 339, 563-572.

[75] Nicot F., Hadda N., Guessasma M., Fortin J., Millet O., (2013), On the definition of the stress tensor in granular media, International Journal of Solids and Structures, 50(14-15), 2508-2517.

[76] Jaeger H. M., Nagel S. R., and Behringer R. P., (1996), Granular solids, liquids, and gases, Rev. Mod. Phys., 68, 1259-1273.

[77] Larson R. G., Shaqfeh E. S. G., and Muller S. J., (1990), A purely elastic instabilityin TaylorCouette flow, J. Fluid Mech., 218, 573-600.

[78] Larson R. G., (1992), Instabilities in viscoelastic flows, Rheol.Acta, 31, 213-263.

[79] Groisman A. and Steinberg V., (1998), Mechanism of elastic instability in Couette flow of polymer solutions, Experiment, Phys Fluids, 10(10), 2451-2463.

[80] Morozov A.N. and van Saarloos W., (2005), Subcritical Finite-Amplitude Solutions for Plane Couette Flow of Viscoelastic Fluids, Phys. Rev. Lett. 95, 024501.

[81] Kraichnan R.H., (1974), On Kolmogorov's inertial-range theories, J. Fluid Mech., 62, 305-330. [82] Groisman A. and Steinberg V., (2000), Elastic turbulence in a polymer solution flow, Nature, 405, 53-55. doi:10.1038/35011019

[83] Khayat R., (1994), Chaos and overstability in the thermal convection of viscoelastic fluids, $J$. Non-Newtonian Fluid Mech. 53, 227-255.

[84] Khayat R., (1995), Nonlinear overstability in the thermal convection of viscoelastic fluids, $J$. Non-Newtonian Fluid Mech. 58, 331-356.

[85] Avila K., Moxey D., de Lozar A., Avila M., Barkley D., Hof B., (2011), The Onset of Turbulence in Pipe Flow, Science 333, 192-196.

[86] Wygnanski I. J. and Champagne F. H., (1973), On transition in a pipe. Part 1.The origin of puffs and slugs and the flow in a turbulent slug, J. Fluid Mech., 59, 281-335. 
[87] Eckhardt B, Schneider T.M., Hof B., Westerweel J., (2007), Turbulence transition in pipe Flow, Annu Rev Fluid Mech 39,447-468.

[88] Kaneko K., (1985), Spatiotemporal Intermittency in Coupled Map Lattices, Progress of Theoretical Physics, 74(5), 1033-1044.

[89] Lappa M., (2006), Oscillatory convective structures and solutal jets originated from discrete distributions of droplets in organic alloys with a miscibility gap, Phys. Fluids,18 (4), 042105 (14 pages).

[90] Lappa M., (2007), "Single- and Multi-droplet configurations out of thermodynamic equilibrium: pulsating, traveling and erratic fluid-dynamic instabilities”, Chapter 1 (pp. 1-58) in New Colloid and Surface Science Research, Editor Lawrence V. Schwartz, 2007 Nova Science Publishers Inc.

[91] Barten W., Lucke M., Kamps M. and Schmitz R., (1995), Convection in binary mixtures. II. Localised traveling waves, Phys. Rev. E 51, 5662-5680.

[92] Pauluis O. and Schumacher J., (2011), Self-aggregation of clouds in conditionally unstable moist convection, PNAS 108 (31) 12623-12628. https://doi.org/10.1073/pnas.1102339108

Smith A.L. and Wensrich C.M., (2014), The effects of particle dynamics on the calculation of bulk stress in granular media, International Journal of Solids and Structures 51, 4414-4418. 